\begin{tabular}{|c|l|}
\hline Title & Molecular structure of cotinine studied by gas electron diffraction combined with theoretical calcul ations \\
\hline Author(s) & Takeshima, Tsuguhide; Takeuchi, Hiroshi; Egawa, Toru; Konaka, Shigehiro \\
\hline Citation & $\begin{array}{l}\text { Journal of Molecular Structure, 841(1-3), 13-21 } \\
\text { https://doi.org/10.1016/.molstruc.2006.11.041 }\end{array}$ \\
\hline Issue Date & $2007-09$-30 \\
\hline Doc URL & http:/hdl.handle.net/2115/28247 \\
\hline Type & article (author version) \\
\hline File Information & JMS841-1-3.pdf \\
\hline
\end{tabular}

Instructions for use 


\title{
Molecular structure of cotinine studied by gas electron diffraction combined with theoretical calculations
}

\author{
Tsuguhide Takeshima ${ }^{1}$, Hiroshi Takeuchi, Toru Egawa ${ }^{*}$ and Shigehiro Konaka \\ Division of Chemistry, Graduate School of Science, Hokkaido University, Sapporo 060-0810, Japan
}

Received:

${ }^{1}$ Present address: Hokkaido Laboratory, Nissei Bio Co. Ltd., Eniwa 061-1374, Japan.

* Corresponding author. Tel.: +81-11-706-3506; fax: +81-11-706-4924.

E-mail address: egawa@sci.hokudai.ac.jp (T. Egawa). 


\section{Abstract}

The molecular structure of cotinine ((S)-1-methyl-5-(3-pyridinyl)-2-pyrrolidinone), the major metabolite of nicotine, has been determined at about $182^{\circ} \mathrm{C}$ by gas electron diffraction combined with MP2 and DFT calculations. The diffraction data are consistent with the existence of the (ax, sc), (ax, ap), (eq, sp) and (eq, ap) conformers, where ax and eq indicate the configuration of the pyrrolidinone ring by means of the position (axial and equatorial) of the pyridine ring, and $s c, s p$ and ap distinguish the isomers arising from the internal rotation around the bond connecting the two rings. The $\left(\mathrm{CH}_{3}\right) \mathrm{NCCC}(\mathrm{N})$ dihedral angles, $\phi$, of the (ax, sc) and (eq, sp) conformers were determined independently to be $158(12)^{\circ}$ and $129(13)^{\circ}$, respectively, where the numbers in parentheses are three times the standard errors, $3 \sigma$. According to the MP2 calculations, the corresponding dihedral angles for the (ax, ap) and (eq, ap) conformers were assumed to differ by $180^{\circ}$ from their syn counterparts. The ratios $x(\mathrm{ax}, s c) / x(\mathrm{ax}$, $a p)$ and $x(\mathrm{eq}, \mathrm{sp}) / x(\mathrm{eq}, a p)$ were taken from the theoretically estimated free energy differences, $\Delta G$, where $x$ is the abundance of the conformer. The resultant abundances of $(\mathrm{ax}, s c),(\mathrm{ax}, a p),(\mathrm{eq}, \mathrm{sp})$ and (eq, ap) conformers are $34(6) \%, 21 \%$ (d.p.), 28\% (d.p.), and $17 \%$ (d.p.), respectively, where d.p. represents dependent parameters.

The determined structural parameters $\left(r_{\mathrm{g}}(\AA)\right.$ and $\left.\angle_{\alpha}\left({ }^{\circ}\right)\right)$ of the most abundant conformer, $(\mathrm{ax}, s c)$, are as follows: $r(\mathrm{~N}-\mathrm{C})_{\text {pyrrol }}=1.463(5) ; r\left(\mathrm{~N}-\mathrm{C}_{\text {methyl }}\right)=1.457(\leftarrow)$; $r(\mathrm{~N}-\mathrm{C}(=\mathrm{O}))=1.384(12) ; r(\mathrm{C}=\mathrm{O})=1.219(5) ;<r(\mathrm{C}-\mathrm{C})_{\text {pyrrol }}>=1.541(3) ; r\left(\mathrm{C}_{\text {pyrrol }}-\mathrm{C}_{\text {pyrid }}\right)$ $=1.521(\leftarrow) ;<r(\mathrm{C}-\mathrm{C})_{\text {pyrid }}>=1.396(2) ;<r(\mathrm{C}-\mathrm{N})_{\text {pyrid }}>=1.343(\leftarrow) ; \angle(\mathrm{CNC})_{\text {pyrrol }}=$ $113.9(11) ; \angle \mathrm{CCC}_{\text {pyrrol }}\left(-\mathrm{C}_{\text {pyrid }}\right)=103.6(\leftarrow) ; \angle \mathrm{NCO}=124.1(13) ; \angle \mathrm{NC}_{\text {pyrrol }} \mathrm{C}_{\text {pyrid }}=$ $113.1(12) ; \angle \mathrm{C}_{\text {pyrrol }} \mathrm{C}_{\text {pyrrol }} \mathrm{C}_{\text {pyrid }}=113.3(\leftarrow) ; \angle(\mathrm{CNC})_{\text {pyrid }}=117.1(2) ;<\angle(\mathrm{NCC})_{\text {pyrid }}>=$ $124.4(\leftarrow) ; \angle \mathrm{C}_{\text {methyl }} \mathrm{NC}(=\mathrm{O})=\angle \mathrm{C}_{\text {methyl }} \mathrm{NC}\left(-\mathrm{C}_{\text {pyrid }}\right)=122.8($ d.p. $) ; \angle \mathrm{NC}(=\mathrm{O}) \mathrm{C}=$ 107.1(d.p.); $\angle \mathrm{NC}_{\text {pyrrol }}\left(-\mathrm{C}_{\text {pyrid }}\right) \mathrm{C}_{\text {pyrrol }}=103.0$ (d.p. $)$ and $\angle \mathrm{CCC}(=\mathrm{O})=105.2($ d.p. $)$, where $\leftarrow$ in the parentheses means that the parameter is bound to the preceding one and $<>$ denote average values. The puckering angle, $\alpha$, of the pyrrolidinone ring is $26(3)^{\circ}$. 
The $\mathrm{N} \cdots \mathrm{N}$ distances of the (ax, sc) and (eq, sp) conformers, which are 4.844(5) and 4.740(5) $\AA$, respectively, are close to that of the most stable conformer of nicotine, 4.885(6) $\AA$ and the corresponding one of arecoline, 4.832(13) $\AA$. It is concluded that the weak nicotinic activity of cotinine cannot be ascribed to such a small difference in the $\mathrm{N} \cdots \mathrm{N}$ distances.

Keywords: Cotinine, Gas electron diffraction, Molecular structure, Theoretical calculations, Nicotinic activity

\section{Introduction}

It has long been stated that the distances between the active sites of agonistic substance of the nicotinic acetylcholine receptor (nAChR) are close to that between the $\mathrm{N}$ and carbonyl $\mathrm{O}$ atoms of acetylcholine [1]. Recently we have determined the molecular structures and conformation of typical nicotinic agonists, nicotine and arecoline, by gas electron diffraction (GED) $[2,3]$. In those studies, it was found that at least one of the stable conformers of nicotine and arecoline has indeed the $\mathrm{N} \cdots \mathrm{N}$ or $\mathrm{N} \cdots \mathrm{O}_{\text {carbonyl }}$ distance close to the $\mathrm{N} \cdots \mathrm{O}_{\text {carbonyl }}$ distance of acetylcholine.

There is another factor ruling the nicotinic activity according to Yamamoto and co-workers who studied the relationship between the activity of nicotine and a number of related compounds and the charge distribution of their $\mathrm{N}$ atoms by means of ${ }^{15} \mathrm{~N}$ NMR [4]. They stated that the agonist binds strongly with the nAChR of vertebrates if the $\mathrm{sp}^{3}$ $\mathrm{N}$ atom such as that in the five membered ring of nicotine has one unit of positive charge because of protonation. On the other hand, if the $\mathrm{N}$ atom is bonded to the electron withdrawing group, it becomes partially positive but cannot be protonated, and consequently, the interaction with the $\mathrm{nAChR}$ of vertebrates becomes weak. On the other hand, no discussion is given on the relationship between the active site distance and the nicotinic activity in Ref. [4]. 
In the present study, the molecular structure and conformation of cotinine ((S)-1-methyl-5-(3-pyridinyl)-2-pyrrolidinone, see Fig. 1) is investigated by GED. Cotinine is the major metabolite of nicotine and is also a nicotinic agonist with much lower affinity for the receptor than most of known agonists [5]. This compound is simultaneously present with nicotine in vivo environment. Although cotinine is not included in the compounds investigated in Ref. [4], it is clearly classified as the weak agonist of the nAChR of vertebrates according to Yamamoto and co-workers [4]. This is because cotinine has the electron withdrawing $\mathrm{C}=\mathrm{O}$ group adjacent to the $\mathrm{N}$ atom and hence the charge is weaker than the monoprotonated $\mathrm{N}$ atom. Therefore it is expected that the structural determination of cotinine will give some information on the factor of the nicotinic activity.

If the $\mathrm{N} \cdots \mathrm{N}$ distance of cotinine is considerably different from those of the corresponding distances of nicotine and arecoline, the weak nicotinic activity is not necessarily ascribed to the difference in the charge of the $\mathrm{N}$ atoms. On the other hand, if the $\mathrm{N} \cdots \mathrm{N}$ distance of cotinine is close to the corresponding distances of nicotine and arecoline, this may support the conclusion by Yamamoto et al.

In the present study, the MP2 and DFT theoretical calculations are performed to assist the data analysis of GED especially in determining the population of conformers with low relative abundances. Determined structural parameters are compared with those of nicotine and arecoline.

\section{Experimental}

The sample with a purity of better than $98 \%$ was purchased from Sigma Chemical Co. and was used without further purification. Electron diffraction patterns were recorded on $8 \times 8$ in. Kodak projector slide plates with an apparatus equipped with an $r^{3}$-sector [6]. 
Two camera distances were used to cover the $s$ range of 2.2 to $33.4 \AA^{-1}$. Because cotinine has very low vapor pressure at room temperature, the nozzle was heated up to about $182{ }^{\circ} \mathrm{C}$. The accelerating voltage of incident electrons was about $37 \mathrm{kV}$. Other experimental conditions are summarized in Table 1. The photographic plates were developed for $4.5 \mathrm{~min}$ in Dektol developer diluted 1:1. The optical densities of the plates were measured as written elsewhere [7]. The experimental intensities leveled by theoretical backgrounds are listed in the Table S1 of Supplementary Material. The scale factor was calibrated to the $r_{\mathrm{a}}(\mathrm{C}=\mathrm{S})$ distance of $\mathrm{CS}_{2}(1.5570 \AA)$ [8].

Elastic atomic scattering factors were calculated as described in Ref. [9], while inelastic ones were taken from Ref. [10]. The experimental molecular scattering intensities are shown in Fig. 2 with the final fitting curves. A diagonal weight matrix was used in the least-squares analysis on the molecular scattering intensities. The weight function was set unity in the medium s region while it was reduced in the small and large $s$ regions by using two gaussian functions [11].

\section{Theoretical calculations}

\subsection{Possible conformers}

The conformation of cotinine is classified by the following two factors. First, as the five-membered (pyrrolidinone) ring has an $\mathrm{sp}^{2}$ atom, it performs the puckering motion like a four-membered ring to make two nonequivalent envelope forms, which are distinguished by means of the position (axial and equatorial) of the pyridine ring. Second, the internal rotation around the $\mathrm{C}-\mathrm{C}$ bond connecting the rings may cause the rotational isomers, $s p$ (or $s c$ ) and $a p$, whose dihedral angles $\left(\mathrm{CH}_{3}\right) \mathrm{NCCC}(\mathrm{N})$ differ by about $180^{\circ}$ from each other. Consequently, the overall conformation of cotinine is expressed as (ax, sc), (ax, ap), (eq, sp) and (eq, ap). These conformers are shown in Fig. 
3 , where the rotational isomers, $s c, s p$ and $a p$, are defined by using the $\mathrm{H}_{18}-\mathrm{C}_{4}-\mathrm{C}_{7}-\mathrm{C}_{8}$ dihedral angle.

\subsection{Geometrical calculations}

GAUSSIAN 98 program [12] was used in all the following calculations. At first, geometries of (ax, sc), (ax, ap), (eq, sp) and (eq, ap) conformers were optimized by using the RHF method with the $6-31 \mathrm{G}^{* *}$ basis set. The geometries of them were further optimized by using the MP2 (frozen core) method with the $6-31 \mathrm{G}^{* *}$ basis set in order to obtain more reliable structural parameters. The conformational energy differences obtained by the RHF and MP2 calculations are compared in Table 2.

Clearly, the additivity of the energy differences between the (ax)- and (eq)conformers and those between the $s c$ (or $s p$ ) and ap conformers holds. First, on going from the (ax) to (eq)-conformers, energy decreases only slightly in the MP2 calculations, while the difference is much larger in the RHF calculations. Second, the energy differences between $s c$ (or $s p$ ) and ap conformers are about $2.4 \mathrm{~kJ} \mathrm{~mol}^{-1}$ according to the MP2 calculations. The potential functions of the internal rotation around the $\mathrm{C}-\mathrm{C}$ bond connecting the rings were obtained for the (ax) and (eq) conformers by a series of $\mathrm{MP} 2 / 6-31 \mathrm{G}^{* *}$ calculations in which the $\mathrm{C}-\mathrm{C}$ torsional angle was fixed at an interval of $30^{\circ}$. The obtained potential functions (not shown) revealed that no minimum exists other than those corresponding to the above mentioned four conformers.

The structural parameters obtained from the MP2 calculations are listed in Table 3. This table shows that the $s c$ (or $s p$ ) and ap conformers have essentially the same structural parameters except for the difference in the dihedral angles, $\mathrm{X}-\mathrm{C}_{4}-\mathrm{C}_{7}-\mathrm{Y}$, that represent the internal rotation of the pyridine ring by $180^{\circ}$. The obtained dihedral angles

suggest that the $\mathrm{N}_{1}, \mathrm{C}_{3}, \mathrm{C}_{4}, \mathrm{C}_{5}$ and $\mathrm{O}_{13}$ atoms are nearly on the same plane. This can be explained as a result of the $\mathrm{sp}^{2}$ character of the $\mathrm{N}_{1}$ and $\mathrm{C}_{3}$ atoms that forces co-planarity 
for these atoms. On the other hand, the $\mathrm{C}_{6}$ atom is positioned out of the plane to reduce the eclipsing of the $\mathrm{C}-\mathrm{H}$ bonds.

The distances between the $\mathrm{O}_{13}$ atom and the $\mathrm{H}$ atom in the methyl group $\left(\mathrm{H}_{20}\right)$ of the four conformers are in the range of $2.40-2.41 \AA$, suggesting the hydrogen bonding between them.

\subsection{Vibrational calculations}

The geometry optimizations and vibrational calculations for these conformers were carried out with the $6-31 \mathrm{G}^{*}$ basis set by using the B3LYP, DFT method, that is more suitable to obtain reliable force constants than the MP2 method. The Cartesian force constants obtained for the (ax, sc), (ax, ap), (eq, sp) and (eq, ap) conformers by the $\mathrm{B} 3 \mathrm{LYP} / 6-31 \mathrm{G}^{*}$ calculations were transformed into the internal force constants, $f_{i j}$. The $f_{i j}$ 's for each conformer were then modified by using the linear scaling formula $f_{i j}$ (scaled) $=c f_{i j}$ (unscaled) [13]. The scale factor, $c$, of 0.9243, which is the square of the scaling factor of 0.9614 for the B3LYP/6-31G* frequency recommended by Scott and Radom [14] was used. The definitions of internal coordinates and the scaled force constants are available as Supplementary Material. The B3LYP/6-31G* energy differences between the four conformers are listed in Table 2.

Free energy differences, $\Delta G$, at $455 \mathrm{~K}$ were estimated by using the energy differences, $\Delta E$, and the rotational constants obtained from the MP2/6-31G** calculations, combined with the vibrational frequencies calculated by the above mentioned scaled DFT force constants. The obtained $\Delta G$ values and the derived abundances are listed in Table 2 .

All these calculations using the results of Gaussian 98 were carried out by a series of computer programs made in our laboratory.

\section{Analysis of electron diffraction data}


For the analysis of electron diffraction data the following assumptions were adopted on the basis of the results of the MP2 ab initio calculations: (1) the ratio of the abundances of the (ax, $s c)$ and (ax, ap), 0.608, and the ratio of the abundances of (eq, $s p)$ and (eq, $a p), 0.584$, were adopted from the above mentioned theoretical $\Delta G$ values; (2) the dihedral angle, $\mathrm{N}_{1}-\mathrm{C}_{4}-\mathrm{C}_{7}-\mathrm{C}_{8}$, of the ap conformer differs from that of the $s c$ (or $s p$ ) conformer by $180^{\circ}$; (3) the dihedral angle, $\mathrm{C}_{4}-\mathrm{N}_{1}-\mathrm{C}_{3}-\mathrm{C}_{5}$ is $0^{\circ}$ and the $\mathrm{O}_{13}$ atom is assumed to be on the $\mathrm{C}_{4} \mathrm{~N}_{1} \mathrm{C}_{3} \mathrm{C}_{5}$ plane; (4) the dihedral angle, $\mathrm{C}_{2}-\mathrm{N}_{1}-\mathrm{C}_{3}-\mathrm{C}_{5}$ is equal to the MP2/6-31G** value; (5) the absolute values of the ring puckering angle $\alpha$ of all the four conformers are equal (see Fig. 4 for the definition of $\alpha$ ); (6) the $\mathrm{C}_{2}-\mathrm{N}_{1}-\mathrm{C}_{3}$ and $\mathrm{C}_{2}-\mathrm{N}_{1}-\mathrm{C}_{4}$ angles are equal; (7) each $\mathrm{H}-\mathrm{C}-\mathrm{H}$ angle in the pyrrolidinone ring bisects the $\mathrm{C}-\mathrm{C}-\mathrm{C}$ angle; (8) the pyridine ring is planar and each $\mathrm{C}-\mathrm{H}$ bond in it bisects the $\mathrm{N}-\mathrm{C}-\mathrm{C}$ or $\mathrm{C}-\mathrm{C}-\mathrm{C}$ angle; (9) the $\mathrm{C}_{4}-\mathrm{C}_{7}$ bond bisects the $\mathrm{C}_{8}-\mathrm{C}_{7}-\mathrm{C}_{12}$ angle; (10) three $\mathrm{N}_{1}-\mathrm{C}_{2}-\mathrm{H}$ angles are equal to $109.5^{\circ}$, which is the average of the corresponding angles obtained by the MP2/6-31G ${ }^{* *}$ calculations; (11) all the $\mathrm{C}-\mathrm{H}$ bond lengths in the pyrrolidinone ring and the methyl group are equal; (12) the $\mathrm{C}-\mathrm{H}$ bond lengths in the pyridine ring are equal, and they are smaller than those in the $N$-methylpyrrolidinone group by $0.007 \AA$; (13) the differences between the similar structural parameters of intra- and inter-conformers are equal to the MP2/6-31G ${ }^{* *}$ values. The free energy differences, $\Delta G$, between $s c$ (or $s p$ ) and ap conformers had to be fixed (assumption (1)) because it is almost impossible to distinguish two conformers caused by the $180^{\circ}$ internal rotation of the pyridine ring precisely [15]. In addition, Table 3 shows that the $M P 2 / 6-31 G^{* *}$ calculations justify assumption (2). Assumption (3) was adopted because the theoretical dihedral angles, $\mathrm{C}_{4}-\mathrm{N}_{1}-\mathrm{C}_{3}-\mathrm{C}_{5}$, are zero within $\pm 7^{\circ}$ (see Table 3). Assumption (5) was adopted because the absolute values of the theoretical puckering angle, $\alpha$, are equal to each other within $1.1^{\circ}$. Table 4 summarizes the adjustable parameters and the constraints.

Mean amplitudes, $l$, and shrinkage corrections [16], $r_{\mathrm{a}}-r_{\alpha}$, were calculated from the scaled force constants by using a program made in our laboratory. They were fixed at 
the calculated ones in the data analysis. The model of small amplitude vibrations was adopted. Table S4 of Supplementary Material lists the mean amplitudes with the corresponding $r_{\mathrm{a}}$ distances. The anharmonicity parameters, $\kappa$, for bonded atom pairs were estimated as described in Ref. [17]. The adjustable parameters listed in Table 4 were determined by a least-squares calculation on the molecular scattering intensities.

\section{Results and discussion}

The results obtained by GED are listed in Tables 2, 5 and 6 . The radial distribution curve is shown in Fig. 5. The absolute values of correlation coefficients among the independent parameters are less than 0.8. Table 2 shows that the determined populations of four conformers are consistent with those derived from the $\Delta G$ values estimated from the MP2 calculations and the scaled force constants of the B3LYP calculations. Table 5 lists the experimental structural parameters ( $r_{\mathrm{g}}$ and $\angle_{\alpha}$ structure) of the most abundant conformer, $(\mathrm{ax}, s c)$, of cotinine and the corresponding ones given by the MP2 calculation ( $r_{\mathrm{e}}$ structure). The differences between the $r_{\mathrm{g}}$ and $r_{\mathrm{e}}$ structures can be estimated using the diatomic approximation [16], $r_{\mathrm{g}}-r_{\mathrm{e}} \approx(3 / 2) a l^{2}$ and assuming the Morse parameter, $a$, to be $2.0 \AA^{-1}$. This term is estimated to be about $0.018 \AA$ for the C-H bonds, $0.007-0.010 \AA$ for other single bonds, $0.006-0.007 \AA$ for the bonds in the pyridine ring and $0.004 \AA$ for the $\mathrm{C}=\mathrm{O}$ bond. Therefore the bond lengths given by the MP2/6-31G** calculations for the single bonds are in agreement with the experimental ones within $3 \sigma$. The calculated $\mathrm{C}=\mathrm{O}$ bond and the bonds in the pyridine ring are longer than the experimental ones by $9 \sigma$. The theoretical bond angles and dihedral angles are in moderate agreement with the experimental ones. The determined dihedral angles $\phi$ $\left(\mathrm{N}_{1}-\mathrm{C}_{4}-\mathrm{C}_{7}-\mathrm{C}_{8}\right)$ of the (ax, sc) and (eq, sp) conformers are $158(12)^{\circ}$ and $129(13)^{\circ}$, respectively. This difference is due to the difference in the axial and equatorial positions of the pyridine ring that influences the steric repulsion in the non-bonded $\mathrm{H}$ atom pairs 
such like $\mathrm{H}_{18} \cdots \mathrm{H}_{22}$ and $\mathrm{H}_{21} \cdots \mathrm{H}_{25}$.

Table 5 lists the structural parameters of the most abundant conformer of nicotine [2]. The $r_{\mathrm{g}}\left(\mathrm{N}_{1}-\mathrm{C}_{3}\right)$ of cotinine is about $0.08 \AA$ shorter than that of nicotine. The short N-C distance is common to the amide group. The considerable differences in the bond angles between the five-membered ring of cotinine and that of nicotine are due to different conformations of the rings (see Fig. 4).

As written in [1], the $\mathrm{N} \cdots \mathrm{N}$ distance in a nicotinic agonist is related to its affinity for the receptor (nAChR). The $\mathrm{N} \cdots \mathrm{N}$ distances of cotinine and nicotine are compared in Table 6. Also the $\mathrm{N} \cdots \mathrm{O}_{\text {carbonyl }}$ distances of arecoline are listed in this table. The $\mathrm{N} \cdots \mathrm{N}$ distances of cotinine have been determined experimentally for the first time in the present study. In nicotine the $\mathrm{N} \cdots \mathrm{N}$ distance of the most stable conformer is 4.885 (6) $\AA$, while that of the next stable conformer is 4.275 (7) $\AA$. The $\mathrm{N} \cdots \mathrm{O}_{\text {carbonyl }}$ distances of the Eq-s-cis and Eq-s-trans conformers of arecoline are 4.832(13) and 4.265(9) A,, respectively [3], and it was concluded that the major conformer with the longer $\mathrm{N} \cdots \mathrm{O}_{\text {carbonyl }}$ distance (Eq-S-cis) has the nicotinic activity. In cotinine the $\mathrm{N} \cdots \mathrm{N}$ distance of the most stable and the next stable conformers were found to be 4.844 (5) and 4.740 (5) $\AA$, respectively, suggesting that the both of these conformers are those with the nicotinic activity.

Although these three molecules have conformers with the $\mathrm{N} \cdots \mathrm{N}$ or $\mathrm{N} \cdots \mathrm{O}$ atom pairs whose distances fall in the range of $4.7-4.9 \AA$, only cotinine has weaker nicotinic activity than other two. Therefore, our conclusion is that the weak nicotinic activity of cotinine cannot be attributed to the difference in the $\mathrm{N} \cdots \mathrm{N}$ or $\mathrm{N} \cdots \mathrm{O}$ distance. As mentioned in the Introduction, other explanation for the nicotinic activity by means of the difference in the charge on the $\mathrm{sp}^{3} \mathrm{~N}$ atom has been proposed [4]. In order to investigate the relationship between the charge distribution and the nicotinic activity, the natural population analyses [18] for cotinine and protonated nicotine were carried out. They provided a negative charge on the $\mathrm{N}_{1}$ atom of these species with that of cotinine slightly 
more negative ( $-0.49 e$ for cotinine and $-0.44 e$ for protonated nicotine). This result seems consistent with what Yamamoto et al. have suggested in Ref. [4] only qualitatively, and it cannot be concluded if such a small difference in the atomic charge explains the difference in the nicotinic activity. Therefore, other explanation such as one by means of a formation of hydrogen bond between the carbonyl $\mathrm{O}$ and methyl $\mathrm{H}$ atoms cannot be ruled out at this stage.

6. Supplementary material

Tables of the leveled total intensities and the backgrounds, the local symmetry coordinates, the scaled force constants, mean amplitudes and $r_{\mathrm{a}}$ distances of the (ax, sc) conformer, the correlation matrix are documented.

\section{Acknowledgments}

We thank the Computing Center of the Institute for Molecular Science, Okazaki National Research Institutes, for the use of the NEC SX-4 computer and the Library program GAUSSIAN 98. 


\section{References}

[1] L. B. Kier, Molecular Orbital Theory in Drug Research, Academic Press, New York, 1971 Chapter 8.

[2] T. Takeshima, R. Fukumoto, T. Egawa, S. Konaka, J. Phys. Chem. A106 (2002) 8734 .

[3] T. Takeshima, H. Takeuchi, T. Egawa, S. Konaka, J. Mol. Struct. 734 (2005) 15.

[4] I. Yamamoto, G. Yabuta, M. Tomizawa, T. Saito, T. Miyamoto, S. Kagabu, J. Pepticide Sci. 20 (1995) 33.

[5] D. J. Anderson, S. P. Arneric, Eur. J. Pharmacol. 253 (1994) 261.

[6] S. Konaka, M. Kimura, 13th Austin Symposium on Gas-Phase Molecular Structure, 12-14 March; The University of Texas, Austin, TX, 12-14 March 1990, S21.

[7] N. Kuze, H. Fujiwara, H. Takeuchi, T. Egawa, S. Konaka, G. Fogarasi, J. Phys. Chem. A103 (1999) 3054.

[8] A. Tsuboyama, A. Murayama, S. Konaka, M. Kimura, J. Mol. Struct. 118 (1984) 351.

[9] M. Kimura, S. Konaka, M. Ogasawara, J. Chem. Phys. 46 (1967) 2599.

[10] C. Tavard, D. Nicolas, M. Rouault, J. Chim. Phys. Phys.-Chim. Biol. 64 (1967) 540.

[11] S. Konaka, M. Kimura, Bull. Chem. Soc. Jpn. 43 (1970) 1693.

[12] M.J. Frisch, G.W. Trucks, H.B. Schlegel, G.E. Scuseria, M.A. Robb, J.R.

Cheeseman, V.G. Zakrzewski, J.A. Montgomery Jr, R.E. Stratmann, J.C. Burant, S. Dapprich, J.M. Millam, A.D. Daniels, K.N. Kudin, M.C. Strain, O. Farkas, J. Tomasi, V. Barone, M. Cossi, R. Cammi, B. Mennucci, C. Pomelli, C. Adamo, S. Clifford, J. Ochterski, G.A. Petersson, P.Y. Ayala, Q. Cui, K. Morokuma, D.K. Malick, A.D. Rabuck, K. Raghavachari, J.B. Foresman, J.Cioslowski, J.V. Ortiz, A.G. Baboul, B.B. Stefanov, G. Liu, A. Liashenko, P. Piskorz, I. Komaromi, R. Gomperts, R.L. Martin, 
D.J. Fox, T. Keith, M. A. Al-Laham, C.Y. Peng, A. Nanayakkara, M. Challacombe, P.M.W. Gill, B. Johnson, W. Chen, M.W. Wong, J.L. Andres, C. Gonzalez, M.

Head-Gordon, E.S. Replogle and J.A. Pople, Gaussian 98, Revision A.9, Gaussian, Inc., Pittsburgh PA, 1998.

[13] J.E. Boggs, in I. Hargittai, M. Hargittai (Eds.). Stereochemical Applications of Gas-phase Electron Diffraction, Part B, VCH, New York, 1988 Chapter 10.

[14] A.P. Scott. L.J. Radom, J. Phys. Chem. 100 (1996) 16502.

[15] H. Kiyono, R. Tatsunami, T. Kurai, H. Takeuchi, T. Egawa, S. Konaka, J. Phys. Chem. A 102 (1998) 1405.

[16] K. Kuchitsu, S.J. Cyvin in S.J. Cyvin (Ed.), Molecular Structures and Vibrations; Elsevier, Amsterdam, 1972 Chapter 12.

[17] K. Kuchitsu, L.S. Bartell, J. Chem. Phys. 35 (1961) 1945.

[18] NBO, Version 3.1, E.D. Glendening, A.E. Reed, J.E. Carpenter, F. Weinhold. 
Table 1

Experimental conditions for gas electron diffraction for cotinine

\begin{tabular}{lll}
\hline & Short & Long \\
\hline Camera distance $(\mathrm{mm})$ & 244.3 & 485.6 \\
Nozzle temperature $(\mathrm{K})$ & 455 & 459 \\
Electron wavelength $(\AA)$ & 0.06334 & 0.06380 \\
Uncertainty in the scale factor $(3 \sigma)(\%)$ & 0.06 & 0.06 \\
$\begin{array}{l}\text { Background pressure during exposure } \\
\left(10^{-6} \text { Torr) }\right.\end{array}$ & 2.5 & 2.9 \\
Beam current $(\mu \mathrm{A})$ & 1.4 & 1.4 \\
Exposure time $(\mathrm{s})$ & $90-123$ & $47-58$ \\
Number of plates used & 3 & 4 \\
Range of $s$ value $\left(\AA^{-1}\right)$ & $6.1-33.4$ & $2.2-17.4$ \\
\hline
\end{tabular}


Table 2

Relative energies $\Delta E$ and $\Delta G$ and estimated abundances $x$ for the possible conformers of cotinine obtained from RHF/6-31G**, MP2/6-31G** and B3LYP/6-31G* calculations and GED

\begin{tabular}{|c|c|c|c|c|c|c|}
\hline \multirow[t]{2}{*}{ Conf. a } & \multicolumn{3}{|c|}{$\Delta E / \mathrm{kJ} \mathrm{mol}^{-1}$} & \multirow[t]{2}{*}{$\Delta G / \mathrm{kJ} \mathrm{mol}^{-1} \mathrm{~b}$} & \multicolumn{2}{|c|}{$x / \%$} \\
\hline & $\mathrm{RHF} / 6-31 \mathrm{G}^{* *}$ & $\mathrm{MP} 2 / 6-31 \mathrm{G}^{* *}$ & B3LYP/6-31G* & & theor. ${ }^{c}$ & GED $^{d}$ \\
\hline$(\operatorname{ax} s c)$ & 2.17 & 0.53 & 2.51 & 0.00 & 34.6 & $34(6)$ \\
\hline$(\operatorname{ax} a p)$ & 4.44 & 2.95 & 4.67 & 1.89 & 21.0 & $21^{\mathrm{e}}$ \\
\hline$(\mathrm{eq}, \mathrm{sp})$ & $0.00^{\mathrm{f}}$ & $0.00 \mathrm{~g}$ & $0.00^{\mathrm{h}}$ & 0.79 & 28.0 & $28^{\mathrm{e}}$ \\
\hline$(\mathrm{eq}, a p)$ & 2.26 & 2.30 & 2.03 & 2.83 & 16.4 & $17^{\mathrm{e}}$ \\
\hline
\end{tabular}

a See Fig. 3 for the definitions of the conformers.

$\mathrm{b}$ Theoretical values. Estimated by using the energy differences, $\Delta E$, and the rotational constants obtained from the MP2/6-31G** calculations, combined with the vibrational frequencies calculated by the scaled B3LYP/6-31G* force constants.

c Estimated from the theoretical $\Delta G$ values at $455 \mathrm{~K}$.

d Number in parentheses is three times the standard error $(3 \sigma)$ referring to the last significant digit.

e Dependent parameters.

${ }^{f}$ Absolute energy is $-569.4719683 E_{\mathrm{h}}$.

g Absolute energy is $-571.3202672 E_{\mathrm{h}}$.

$\mathrm{h}$ Absolute energy is $-573.0331144 E_{\mathrm{h}}$. 
Table 3

Geometrical parameters of the (ax, sc), (ax, ap), (eq, sp) and (eq, ap) conformers of cotinine obtained from the MP2(frozen core) $/ 6-31 \mathrm{G}^{* *}$ calculations a

\begin{tabular}{|c|c|c|c|c|}
\hline Parameters & $(\mathrm{ax}, \mathrm{sc})$ & $(\mathrm{ax}, a p)$ & $(\mathrm{eq}, \mathrm{sp})$ & $(\mathrm{eq}, a p)$ \\
\hline \multicolumn{5}{|c|}{ Bond lengths $(\AA)$} \\
\hline $\mathrm{N}_{1}-\mathrm{C}_{2}$ & 1.446 & 1.446 & 1.448 & 1.448 \\
\hline $\mathrm{N}_{1}-\mathrm{C}_{3}$ & 1.376 & 1.377 & 1.380 & 1.380 \\
\hline $\mathrm{N}_{1}-\mathrm{C}_{4}$ & 1.451 & 1.450 & 1.456 & 1.455 \\
\hline $\mathrm{C}_{3}-\mathrm{C}_{5}$ & 1.523 & 1.523 & 1.522 & 1.522 \\
\hline $\mathrm{C}_{4}-\mathrm{C}_{6}$ & 1.546 & 1.546 & 1.542 & 1.542 \\
\hline $\mathrm{C}_{5}-\mathrm{C}_{6}$ & 1.527 & 1.527 & 1.526 & 1.526 \\
\hline $\mathrm{C}_{3}=\mathrm{O}_{13}$ & 1.228 & 1.228 & 1.227 & 1.227 \\
\hline $\mathrm{C}_{4}-\mathrm{C}_{7}$ & 1.513 & 1.513 & 1.503 & 1.504 \\
\hline $\mathrm{C}_{8}-\mathrm{N}_{9}$ & 1.343 & 1.345 & 1.344 & 1.343 \\
\hline $\mathrm{N}_{9}-\mathrm{C}_{10}$ & 1.345 & 1.344 & 1.344 & 1.346 \\
\hline $\mathrm{C}_{7}-\mathrm{C}_{8}$ & 1.400 & 1.400 & 1.398 & 1.401 \\
\hline $\mathrm{C}_{10}-\mathrm{C}_{11}$ & 1.395 & 1.396 & 1.397 & 1.395 \\
\hline $\mathrm{C}_{11}-\mathrm{C}_{12}$ & 1.393 & 1.392 & 1.391 & 1.394 \\
\hline $\mathrm{C}_{12}-\mathrm{C}_{7}$ & 1.398 & 1.398 & 1.399 & 1.396 \\
\hline $\mathrm{C}_{2}-\mathrm{H}_{19}$ & 1.090 & 1.090 & 1.093 & 1.093 \\
\hline $\mathrm{C}_{2}-\mathrm{H}_{20}$ & 1.087 & 1.087 & 1.087 & 1.087 \\
\hline $\mathrm{C}_{2}-\mathrm{H}_{21}$ & 1.092 & 1.092 & 1.089 & 1.089 \\
\hline $\mathrm{C}_{4}-\mathrm{H}_{18}$ & 1.095 & 1.096 & 1.101 & 1.101 \\
\hline $\mathrm{C}_{5}-\mathrm{H}_{14}$ & 1.088 & 1.088 & 1.092 & 1.092 \\
\hline $\mathrm{C}_{5}-\mathrm{H}_{15}$ & 1.092 & 1.092 & 1.088 & 1.088 \\
\hline $\mathrm{C}_{6}-\mathrm{H}_{16}$ & 1.090 & 1.091 & 1.089 & 1.089 \\
\hline
\end{tabular}




\begin{tabular}{|c|c|c|c|c|}
\hline $\mathrm{C}_{6}-\mathrm{H}_{17}$ & 1.089 & 1.089 & 1.091 & 1.09 \\
\hline $\mathrm{C}_{8}-\mathrm{H}_{22}$ & 1.087 & 1.085 & 1.086 & 1.08 \\
\hline $\mathrm{C}_{10}-\mathrm{H}_{23}$ & 1.084 & 1.084 & 1.084 & 1.08 \\
\hline $\mathrm{C}_{11}-\mathrm{H}_{24}$ & 1.082 & 1.082 & 1.082 & 1.08 \\
\hline $\mathrm{C}_{12}-\mathrm{H}_{25}$ & 1.084 & 1.085 & 1.084 & \\
\hline $\mathrm{N}_{1} \cdots \mathrm{N}_{9}$ & 4.859 & 4.224 & 4.795 & 4.24 \\
\hline \multicolumn{5}{|c|}{ Bond angles (degree) } \\
\hline $\mathrm{C}_{2}-\mathrm{N}_{1}-\mathrm{C}_{3}$ & 122.3 & 122.3 & 121.2 & 121.1 \\
\hline $\mathrm{C}_{2}-\mathrm{N}_{1}-\mathrm{C}_{4}$ & 122.4 & 122.5 & 121.7 & 121.8 \\
\hline $\mathrm{C}_{3}-\mathrm{N}_{1}-\mathrm{C}_{4}$ & 114.0 & 114.1 & 113.4 & 113.3 \\
\hline $\mathrm{N}_{1}-\mathrm{C}_{3}-\mathrm{C}_{5}$ & 107.0 & 106.9 & 107.1 & 107.2 \\
\hline $\mathrm{N}_{1}-\mathrm{C}_{3}=\mathrm{O}_{13}$ & 125.6 & 125.6 & 125.5 & 125.5 \\
\hline $\mathrm{C}_{5}-\mathrm{C}_{3}=\mathrm{O}_{13}$ & 127.4 & 127.5 & 127.3 & 127.3 \\
\hline $\mathrm{N}_{1}-\mathrm{C}_{4}-\mathrm{C}_{6}$ & 101.9 & 101.9 & 102.0 & 102.0 \\
\hline $\mathrm{N}_{1}-\mathrm{C}_{4}-\mathrm{C}_{7}$ & 112.6 & 113.1 & 112.3 & 112.6 \\
\hline $\mathrm{C}_{6}-\mathrm{C}_{4}-\mathrm{C}_{7}$ & 112.9 & 112.8 & 113.4 & 113.4 \\
\hline $\mathrm{C}_{3}-\mathrm{C}_{5}-\mathrm{C}_{6}$ & 104.1 & 104.0 & 104.1 & 104.1 \\
\hline $\mathrm{C}_{5}-\mathrm{C}_{6}-\mathrm{C}_{4}$ & 103.7 & 103.7 & 103.9 & 103.8 \\
\hline $\mathrm{C}_{4}-\mathrm{C}_{7}-\mathrm{C}_{8}$ & 120.0 & 121.9 & 121.0 & 120.6 \\
\hline $\mathrm{C}_{4}-\mathrm{C}_{7}-\mathrm{C}_{12}$ & 122.2 & 120.5 & 121.1 & 121.5 \\
\hline $\mathrm{C}_{8}-\mathrm{C}_{7}-\mathrm{C}_{12}$ & 117.7 & 117.6 & 117.9 & 117.9 \\
\hline $\mathrm{C}_{7}-\mathrm{C}_{8}-\mathrm{N}_{9}$ & 124.5 & 124.1 & 124.3 & 124.1 \\
\hline $\mathrm{C}_{8}-\mathrm{N}_{9}-\mathrm{C}_{10}$ & 116.7 & 117.0 & 116.7 & 116.9 \\
\hline $\mathrm{N}_{9}-\mathrm{C}_{10}-\mathrm{C}_{11}$ & 123.5 & 123.5 & 123.6 & 123.5 \\
\hline $\mathrm{C}_{10}-\mathrm{C}_{11}-\mathrm{C}_{12}$ & 118.9 & 118.6 & 118.8 & 118.7 \\
\hline $\mathrm{C}_{11}-\mathrm{C}_{12}-\mathrm{C}_{7}$ & 118.7 & 119.2 & 118.7 & 118.9 \\
\hline $\mathrm{N}_{1}-\mathrm{C}_{2}-\mathrm{H}_{19}$ & 110.3 & 110.4 & 110.9 & 110.8 \\
\hline
\end{tabular}




\begin{tabular}{|c|c|c|c|c|}
\hline $\mathrm{N}_{1}-\mathrm{C}_{2}-\mathrm{H}_{20}$ & 107.2 & 107.2 & 107.2 & 107.1 \\
\hline $\mathrm{N}_{1}-\mathrm{C}_{2}-\mathrm{H}_{21}$ & 111.1 & 111.1 & 110.6 & 110.6 \\
\hline $\mathrm{H}_{19}-\mathrm{C}_{2}-\mathrm{H}_{20}$ & 109.8 & 109.8 & 109.4 & 109.4 \\
\hline $\mathrm{H}_{19}-\mathrm{C}_{2}-\mathrm{H}_{21}$ & 108.9 & 109.0 & 108.9 & 108.9 \\
\hline $\mathrm{H}_{20}-\mathrm{C}_{2}-\mathrm{H}_{21}$ & 109.5 & 109.5 & 109.9 & 109.9 \\
\hline $\mathrm{N}_{1}-\mathrm{C}_{4}-\mathrm{H}_{18}$ & 109.8 & 109.7 & 110.1 & 110.0 \\
\hline $\mathrm{C}_{6}-\mathrm{C}_{4}-\mathrm{H}_{18}$ & 110.8 & 110.8 & 110.2 & 110.1 \\
\hline $\mathrm{C}_{7}-\mathrm{C}_{4}-\mathrm{H}_{18}$ & 108.7 & 108.5 & 108.7 & 108.5 \\
\hline $\mathrm{C}_{3}-\mathrm{C}_{5}-\mathrm{H}_{14}$ & 110.4 & 110.4 & 107.5 & 107.5 \\
\hline $\mathrm{C}_{3}-\mathrm{C}_{5}-\mathrm{H}_{15}$ & 108.1 & 108.0 & 110.8 & 110.7 \\
\hline $\mathrm{H}_{14}-\mathrm{C}_{5}-\mathrm{H}_{15}$ & 107.7 & 107.8 & 107.7 & 107.8 \\
\hline $\mathrm{C}_{6}-\mathrm{C}_{5}-\mathrm{H}_{14}$ & 114.3 & 114.3 & 111.9 & 111.9 \\
\hline $\mathrm{C}_{6}-\mathrm{C}_{5}-\mathrm{H}_{15}$ & 112.1 & 112.1 & 114.6 & 114.6 \\
\hline $\mathrm{C}_{5}-\mathrm{C}_{6}-\mathrm{H}_{16}$ & 110.0 & 110.0 & 114.3 & 114.3 \\
\hline $\mathrm{C}_{5}-\mathrm{C}_{6}-\mathrm{H}_{17}$ & 114.1 & 114.0 & 110.4 & 110.4 \\
\hline $\mathrm{H}_{16}-\mathrm{C}_{6}-\mathrm{H}_{17}$ & 108.5 & 108.5 & 108.1 & 108.2 \\
\hline $\mathrm{C}_{4}-\mathrm{C}_{6}-\mathrm{H}_{16}$ & 108.7 & 108.8 & 111.5 & 111.5 \\
\hline $\mathrm{C}_{4}-\mathrm{C}_{6}-\mathrm{H}_{17}$ & 111.7 & 111.7 & 108.5 & 108.5 \\
\hline $\mathrm{C}_{7}-\mathrm{C}_{8}-\mathrm{H}_{22}$ & 119.8 & 120.2 & 119.8 & 119.7 \\
\hline $\mathrm{N}_{9}-\mathrm{C}_{8}-\mathrm{H}_{22}$ & 115.7 & 115.7 & 115.9 & 116.2 \\
\hline $\mathrm{N}_{9}-\mathrm{C}_{10}-\mathrm{H}_{23}$ & 115.9 & 115.9 & 115.9 & 115.9 \\
\hline $\mathrm{C}_{11}-\mathrm{C}_{10}-\mathrm{H}_{23}$ & 120.6 & 120.6 & 120.5 & 120.6 \\
\hline $\mathrm{C}_{10}-\mathrm{C}_{11}-\mathrm{H}_{24}$ & 120.1 & 120.3 & 120.1 & 120.2 \\
\hline $\mathrm{C}_{12}-\mathrm{C}_{11}-\mathrm{H}_{24}$ & 120.9 & 121.1 & 121.1 & 121.1 \\
\hline $\mathrm{C}_{11}-\mathrm{C}_{12}-\mathrm{H}_{25}$ & 120.7 & 120.7 & 121.4 & 120.9 \\
\hline $\mathrm{C}_{7}-\mathrm{C}_{12}-\mathrm{H}_{25}$ & 120.5 & 120.1 & 119.9 & 120.2 \\
\hline$\alpha^{\mathrm{b}}$ & 28.2 & 28.9 & -27.9 & -27.8 \\
\hline
\end{tabular}


Dihedral angles (degree)

\begin{tabular}{|c|c|c|c|c|}
\hline $\mathrm{C}_{5}-\mathrm{C}_{3}-\mathrm{N}_{1}-\mathrm{C}_{4}$ & 5.5 & 4.2 & -6.7 & -7.0 \\
\hline $\mathrm{C}_{6}-\mathrm{C}_{5}-\mathrm{C}_{3}-\mathrm{N}_{1}$ & 14.4 & 15.6 & -13.4 & -13.2 \\
\hline $\mathrm{C}_{4}-\mathrm{C}_{6}-\mathrm{C}_{5}-\mathrm{C}_{3}$ & -27.2 & -27.9 & 26.9 & 26.9 \\
\hline $\mathrm{N}_{1}-\mathrm{C}_{4}-\mathrm{C}_{6}-\mathrm{C}_{5}$ & 29.7 & 29.7 & -30.1 & -30.3 \\
\hline $\mathrm{C}_{3}-\mathrm{N}_{1}-\mathrm{C}_{4}-\mathrm{C}_{6}$ & -22.6 & -21.7 & 23.5 & 23.8 \\
\hline $\mathrm{C}_{2}-\mathrm{N}_{1}-\mathrm{C}_{4}-\mathrm{C}_{6}$ & 170.6 & 170.3 & -178.0 & -177.7 \\
\hline $\mathrm{C}_{5}-\mathrm{C}_{3}-\mathrm{N}_{1}-\mathrm{C}_{2}$ & 172.4 & 172.3 & -165.3 & -165.6 \\
\hline $\mathrm{O}_{13}=\mathrm{C}_{3}-\mathrm{N}_{1}-\mathrm{C}_{2}$ & -6.3 & -6.4 & 13.3 & 12.9 \\
\hline $\mathrm{O}_{13}=\mathrm{C}_{3}-\mathrm{N}_{1}-\mathrm{C}_{4}$ & -173.2 & -174.5 & 171.9 & 171.5 \\
\hline $\mathrm{C}_{6}-\mathrm{C}_{5}-\mathrm{C}_{3}=\mathrm{O}_{13}$ & -166.9 & -165.8 & 168.0 & 168.3 \\
\hline $\mathrm{C}_{3}-\mathrm{N}_{1}-\mathrm{C}_{4}-\mathrm{C}_{7}$ & 98.7 & 99.7 & 145.3 & 145.7 \\
\hline $\mathrm{C}_{5}-\mathrm{C}_{6}-\mathrm{C}_{4}-\mathrm{C}_{7}$ & -91.3 & -91.9 & -151.1 & -151.7 \\
\hline $\mathrm{N}_{1}-\mathrm{C}_{4}-\mathrm{C}_{7}-\mathrm{C}_{8}$ & 151.9 & -29.0 & 137.0 & -43.7 \\
\hline $\mathrm{C}_{6}-\mathrm{C}_{4}-\mathrm{C}_{7}-\mathrm{C}_{8}$ & -93.3 & 86.0 & -108.0 & 71.5 \\
\hline $\mathrm{N}_{1}-\mathrm{C}_{4}-\mathrm{C}_{7}-\mathrm{C}_{12}$ & -30.6 & 153.7 & -45.0 & 138.6 \\
\hline $\mathrm{C}_{6}-\mathrm{C}_{4}-\mathrm{C}_{7}-\mathrm{C}_{12}$ & 84.2 & -91.3 & 70.1 & -106.2 \\
\hline $\mathrm{H}_{18}-\mathrm{C}_{4}-\mathrm{C}_{7}-\mathrm{C}_{8}$ & 30.1 & -150.9 & 14.9 & -165.8 \\
\hline
\end{tabular}

a See Fig. 3 for the atom numbering and the definitions of the conformers.

b See Fig. 4 for the definition. 
Table 4

Structural parameters and constraints for the (ax, sc), (ax, ap), (eq, sp) and (eq, ap) conformers of cotinine ${ }^{a}$

\begin{tabular}{|c|c|c|c|c|}
\hline Parameter & $(\mathrm{ax}, \mathrm{sc})$ & $(\mathrm{ax}, a p)$ & $(\mathrm{eq}, \mathrm{sp})$ & $(\mathrm{eq}, a p)$ \\
\hline \multicolumn{5}{|c|}{ Bond lengths ( $\AA)$} \\
\hline $\mathrm{N}_{1}-\mathrm{C}_{2}$ & $r_{1}$ & $r_{1}$ & $r_{1}+0.002$ & $r_{1}+0.002$ \\
\hline $\mathrm{N}_{1}-\mathrm{C}_{4}$ & $r_{1}+0.005$ & $r_{1}+0.005$ & $r_{1}+0.010$ & $r_{1}+0.010$ \\
\hline $\mathrm{N}_{1}-\mathrm{C}_{3}$ & $r_{2}$ & $r_{2}+0.001$ & $r_{2}+0.004$ & $r_{2}+0.004$ \\
\hline $\mathrm{C}_{3}-\mathrm{C}_{5}$ & $r_{3}$ & $r_{3}$ & $r_{3}-0.001$ & $r_{3}-0.001$ \\
\hline $\mathrm{C}_{4}-\mathrm{C}_{6}$ & $r_{3}+0.023$ & $r_{3}+0.023$ & $r_{3}+0.019$ & $r_{3}+0.019$ \\
\hline $\mathrm{C}_{5}-\mathrm{C}_{6}$ & $r_{3}+0.004$ & $r_{3}+0.004$ & $r_{3}+0.003$ & $r_{3}+0.003$ \\
\hline $\mathrm{C}_{4}-\mathrm{C}_{7}$ & $r_{3}-0.011$ & $r_{3}-0.010$ & $r_{3}-0.020$ & $r_{3}-0.019$ \\
\hline $\mathrm{C}_{3}=\mathrm{O}_{13}$ & $r_{4}$ & $r_{4}-0.001$ & $r_{4}-0.001$ & $r_{4}-0.001$ \\
\hline $\mathrm{C}_{8}-\mathrm{N}_{9}$ & $r_{5}$ & $r_{5}+0.002$ & $r_{5}+0.001$ & $r_{5}$ \\
\hline $\mathrm{N}_{9}-\mathrm{C}_{10}$ & $r_{5}+0.003$ & $r_{5}+0.001$ & $r_{5}+0.001$ & $r_{5}+0.003$ \\
\hline $\mathrm{C}_{7}-\mathrm{C}_{8}$ & $r_{5}+0.057$ & $r_{5}+0.058$ & $r_{5}+0.055$ & $r_{5}+0.059$ \\
\hline $\mathrm{C}_{10}-\mathrm{C}_{11}$ & $r_{5}+0.052$ & $r_{5}+0.053$ & $r_{5}+0.054$ & $r_{5}+0.052$ \\
\hline $\mathrm{C}_{11}-\mathrm{C}_{12}$ & $r_{5}+0.051$ & $r_{5}+0.049$ & $r_{5}+0.049$ & $r_{5}+0.051$ \\
\hline $\mathrm{C}_{7}-\mathrm{C}_{12}$ & $r_{5}+0.055$ & $r_{5}+0.055$ & $r_{5}+0.056$ & $r_{5}+0.053$ \\
\hline $\mathrm{C}-\mathrm{H}_{\text {pyrro }}$ & $r_{6}$ & $r_{6}$ & $r_{6}$ & $r_{6}$ \\
\hline $\mathrm{C}-\mathrm{H}_{\text {pyrid }}$ & $r_{6}-0.007$ & $r_{6}-0.007$ & $r_{6}-0.007$ & $r_{6}-0.007$ \\
\hline
\end{tabular}

Bond angles (degree) 


\begin{tabular}{|c|c|c|c|c|}
\hline $\mathrm{C}_{3}-\mathrm{N}_{1}-\mathrm{C}_{4}$ & $\theta_{1}$ & $\theta_{1}+0.1$ & $\theta_{1}-0.6$ & $\theta_{1}-0.6$ \\
\hline $\mathrm{C}_{4}-\mathrm{C}_{6}-\mathrm{C}_{5}$ & $\theta_{1}-10.2$ & $\theta_{1}-10.3$ & $\theta_{1}-10.1$ & $\theta_{1}-10.2$ \\
\hline$\alpha^{\mathrm{b}}$ & $\theta_{2}$ & $\theta_{2}$ & $-\theta_{2}$ & $-\theta_{2}$ \\
\hline $\mathrm{C}-\mathrm{C}-\mathrm{H}_{\text {methyl }}$ & 109.5 & 109.5 & 109.5 & 109.5 \\
\hline $\mathrm{H}-\mathrm{C}-\mathrm{H}$ methylene & $\theta_{3}$ & $\theta_{3}$ & $\theta_{3}$ & $\theta_{3}$ \\
\hline $\mathrm{N}_{1}-\mathrm{C}_{4}-\mathrm{C}_{7}$ & $\theta_{4}$ & $\theta_{4}+0.4$ & $\theta_{4}-0.3$ & $\theta_{4}$ \\
\hline $\mathrm{C}_{6}-\mathrm{C}_{4}-\mathrm{C}_{7}$ & $\theta_{4}+0.3$ & $\theta_{4}+0.2$ & $\theta_{4}+0.8$ & $\theta_{4}+0.7$ \\
\hline $\mathrm{C}_{8}-\mathrm{N}_{9}-\mathrm{C}_{10}$ & $\theta_{5}$ & $\theta_{5}+0.3$ & $\theta_{5}+0.1$ & $\theta_{5}+0.2$ \\
\hline $\mathrm{C}_{7}-\mathrm{C}_{8}-\mathrm{N}_{9}$ & $\theta_{5}+7.8$ & $\theta_{5}+7.4$ & $\theta_{5}+7.6$ & $\theta_{5}+7.4$ \\
\hline $\mathrm{N}_{9}-\mathrm{C}_{10}-\mathrm{C}_{11}$ & $\theta_{5}+6.8$ & $\theta_{5}+6.8$ & $\theta_{5}+6.9$ & $\theta_{5}+6.9$ \\
\hline $\mathrm{N}_{1}-\mathrm{C}_{3}=\mathrm{O}_{13}$ & $\theta_{6}$ & $\theta_{6}$ & $\theta_{6}-0.1$ & $\theta_{6}$ \\
\hline
\end{tabular}

Dihedral angles (degree)

\begin{tabular}{ccccc}
$\mathrm{N}_{1}-\mathrm{C}_{4}-\mathrm{C}_{7}-\mathrm{C}_{8}$ & $\phi_{1}$ & $\phi_{1}+180.0$ & $\phi_{2}$ & $\phi_{2}+180.0$ \\
$\mathrm{C}_{2}-\mathrm{N}_{1}-\mathrm{C}_{3}-\mathrm{C}_{5}$ & 172.4 & 172.3 & -165.3 & -165.6 \\
\hline
\end{tabular}

a See Fig. 3 for the atom numbering and the definitions of the conformers.

b See Fig. 4 for the definition. 
Table 5

Molecular structures of cotinine and nicotine

\begin{tabular}{lll}
\hline & Cotinine $^{\mathrm{b}}$ & Nicotine $^{\mathrm{c}}$ \\
Parameters $^{\mathrm{a}}$ & $(\mathrm{ax}, \mathrm{sc})$ & $($ eq, eq, syn $)$ \\
\cline { 2 - 3 } & $\mathrm{ED}\left(r_{\mathrm{g}} \text { and } \angle_{\alpha}\right)^{\mathrm{d}}{\mathrm{MP} 2 / 6-31 \mathrm{G}^{* *}\left(r_{\mathrm{e}}\right)}^{\mathrm{ED}\left(r_{\mathrm{g}} \text { and } \angle_{\alpha}\right)^{\mathrm{d}}}$ & \\
\hline
\end{tabular}

Bond lengths ( $\AA$ )

$\mathrm{N}_{1}-\mathrm{C}_{2}$

1.457

1.446

1.456

$\mathrm{N}_{1}-\mathrm{C}_{4}$

$1.463\}$

(5)

$\mathrm{N}_{1}-\mathrm{C}_{3}$

$1.384 \quad$ (12)

1.451

1.467

$\mathrm{C}_{3}-\mathrm{C}_{5}$

1.532

1.376

1.464

$\mathrm{C}_{4}-\mathrm{C}_{6}$

1.555

$\mathrm{C}_{5}-\mathrm{C}_{6}$

1.536

(3)

1.523

1.546

1.534

1.541

1.527

1.547

$\mathrm{C}_{4}-\mathrm{C}_{7}$

1.521

1.513

1.502

$\mathrm{C}_{3}=\mathrm{O}_{13}$

1.219

(5)

1.228

$\mathrm{C}_{8}-\mathrm{N}_{9}$

1.342

1.343

$\mathrm{C}_{10}-\mathrm{N}_{9}$

1.344

1.345

1.354

$\mathrm{C}_{7}-\mathrm{C}_{8}$

1.399

(2)

1.400

1.344

$\mathrm{C}_{10}-\mathrm{C}_{11}$

1.394

1.395

1.398

(3)

$\mathrm{C}_{11}-\mathrm{C}_{12}$

1.393

1.393

1.397

$\mathrm{C}_{7}-\mathrm{C}_{12}$

1.397

1.398

1.392

$\mathrm{C}-\mathrm{H}_{\text {pyrrol }}$

1.109

$1.091^{\mathrm{e}}$

$\mathrm{C}-\mathrm{H}_{\text {pyrid }}$

1.102

(5)

$1.084^{\mathrm{e}}$

1.399

1.116

1.116

Bond angles and dihedral angles (degree)
$\mathrm{C}_{3}-\mathrm{N}_{1}-\mathrm{C}_{4}$
113.9 )
114.0
$108.4(15)$
$\mathrm{C}_{4}-\mathrm{C}_{6}-\mathrm{C}_{5}$
103.6
$\zeta(11)$
103.7
$108.6^{\mathrm{f}}$
$\mathrm{C}_{2}-\mathrm{N}_{1}-\mathrm{C}_{3}$
$122.8^{\mathrm{f}}$
122.3
$114.1^{\mathrm{f}}$ 


\begin{tabular}{|c|c|c|c|c|}
\hline $\mathrm{C}_{2}-\mathrm{N}_{1}-\mathrm{C}_{4}$ & $122.8^{\mathrm{f}}$ & & 122.4 & $114.1^{\mathrm{f}}$ \\
\hline $\mathrm{N}_{1}-\mathrm{C}_{3}-\mathrm{C}_{5}$ & $107.1 \mathrm{f}$ & & 107.0 & $103.4^{f}$ \\
\hline $\mathrm{N}_{1}-\mathrm{C}_{3}=\mathrm{O}_{13}$ & 124.1 & (13) & 125.6 & \\
\hline $\mathrm{N}_{1}-\mathrm{C}_{4}-\mathrm{C}_{6}$ & $103.0 \mathrm{f}$ & & 101.9 & $101.3^{f}$ \\
\hline $\mathrm{N}_{1}-\mathrm{C}_{4}-\mathrm{C}_{7}$ & 113.1 & Y & 112.6 & $116.8(10)$ \\
\hline $\mathrm{C}_{6}-\mathrm{C}_{4}-\mathrm{C}_{7}$ & 113.3 & & 112.9 & $115.8(20)$ \\
\hline $\mathrm{C}_{3}-\mathrm{C}_{5}-\mathrm{C}_{6}$ & $105.2 \mathrm{f}$ & & 104.1 & $107.7 \mathrm{f}$ \\
\hline $\mathrm{C}_{8}-\mathrm{N}_{9}-\mathrm{C}_{10}$ & 117.1 & ) & 116.7 & 116.8 \\
\hline $\mathrm{C}_{7}-\mathrm{C}_{8}-\mathrm{N}_{9}$ & 124.9 & (2) & 124.5 & 124.5 \\
\hline $\mathrm{N}_{9}-\mathrm{C}_{10}-\mathrm{C}_{11}$ & 123.9 & & 123.5 & 123.7 \\
\hline $\mathrm{C}_{8}-\mathrm{C}_{7}-\mathrm{C}_{12}$ & $115.8 \mathrm{f}$ & & 117.7 & $117.1^{\mathrm{f}}$ \\
\hline $\mathrm{C}_{10}-\mathrm{C}_{11}-\mathrm{C}_{12}$ & $117.4 \mathrm{f}$ & & 118.9 & $118.3^{f}$ \\
\hline $\mathrm{C}_{7}-\mathrm{C}_{12}-\mathrm{C}_{11}$ & $120.9^{f}$ & & 118.7 & $119.5^{f}$ \\
\hline $\mathrm{H}-\mathrm{C}-\mathrm{H}_{\text {methylene }}$ & 108.4 & (57) & $108.1 \mathrm{e}$ & $111.9(41)$ \\
\hline $\mathrm{N}_{1}-\mathrm{C}_{2}-\mathrm{H}$ & 109.5 & (fix) & $109.5^{\mathrm{e}}$ & 113.5 (fix) \\
\hline$\alpha^{\mathrm{g}}$ & 26.4 & (33) & 28.2 & $32.9(38)$ \\
\hline $\mathrm{C}_{6}-\mathrm{C}_{4}-\mathrm{C}_{7}-\mathrm{C}_{8} \mathrm{~h}$ & $-86^{f}$ & & -93.3 & $-87.7(74)$ \\
\hline $\mathrm{N}_{1}-\mathrm{C}_{4}-\mathrm{C}_{7}-\mathrm{C}_{8} \mathrm{~h}$ & 158 & (12) & 151.9 & $153.1^{\mathrm{f}}$ \\
\hline $\mathrm{C}_{6}-\mathrm{C}_{4}-\mathrm{C}_{7}-\mathrm{C}_{8} \mathrm{i}$ & $-114 \mathrm{f}$ & & -108.0 & \\
\hline $\mathrm{N}_{1}-\mathrm{C}_{4}-\mathrm{C}_{7}-\mathrm{C}_{8} \mathrm{i}$ & 129 & (13) & 137.0 & \\
\hline
\end{tabular}

Indices of resolution $\mathrm{j}$

\begin{tabular}{lll}
$k$ (long) & 0.941 & $(12)$ \\
$k$ (short) & 0.901 & $(22)$ \\
\hline
\end{tabular}

a See Fig. 3 for the atom numberings. Angle brackets denote average values.

b Present work. See Fig. 3 for the definitions of the conformers. c See Ref. [2]. 
d Numbers in parentheses are three times the standard errors $(3 \sigma)$ referring to the last significant digit. e Average values ${ }^{f}$ Dependent parameters. $g$ Defined as the dihedral angle between the $\mathrm{C}_{4} \mathrm{C}_{3} \mathrm{C}_{5}$ and $\mathrm{C}_{4} \mathrm{C}_{6} \mathrm{C}_{5}$ planes (see Fig. 4). $\mathrm{h}$ (ax, sc) conformer. $\quad \mathrm{i}$ (eq, $s p$ ) conformer. $\quad \mathrm{j}$ The index of resolution, $k$, is defined as $s M(s)^{\mathrm{obs}}=$ $k s M(s)^{\text {calc. }}$. 
Table 6

The $\mathrm{N} \cdots \mathrm{N}$ or $\mathrm{N} \cdots \mathrm{O}_{\text {carbonyl }}$ distance and the abundance $x$ of each conformer of cotinine, nicotine and arecoline.

\begin{tabular}{|c|c|c|c|c|c|c|c|c|}
\hline \multicolumn{3}{|c|}{ Cotinine ${ }^{a}$} & \multicolumn{3}{|l|}{ Nicotine $b$} & \multicolumn{3}{|l|}{ Arecoline $\mathrm{c}$} \\
\hline Conf. ${ }^{d}$ & $\mathrm{~N} \cdots \mathrm{N} / \AA$ & $x / \%$ & Conf. & $\mathrm{N} \cdots \mathrm{N} / \AA$ & $x / \%$ & Conf. & $\mathrm{N} \cdots \mathrm{O} / \AA \AA \mathrm{e}$ & $x / \%$ \\
\hline$(\mathrm{ax}, \mathrm{sc})$ & $4.844(5)$ & $34(6)$ & $(e q, e q$, syn $)$ & $4.885(6)$ & $67 \mathrm{f}$ & Eq-s-cis & $4.832(13)$ & $46(16)$ \\
\hline$(\mathrm{ax}, a p)$ & $4.198(5)$ & $21 \mathrm{~g}$ & (eq, eq, anti) & $4.275(7)$ & $33 \mathrm{f}$ & Eq-s-trans & $4.265(9)$ & $41 \mathrm{~g}$ \\
\hline$(\mathrm{eq}, \mathrm{sp})$ & $4.740(5)$ & $28 \mathrm{~g}$ & & & & Ax-s-cis & $4.874(16)$ & $7 \mathrm{f}$ \\
\hline$(\mathrm{eq}, a p)$ & $4.299(5)$ & $17 \mathrm{~g}$ & & & & Ax-s-trans & $4.289(12)$ & $6^{f}$ \\
\hline
\end{tabular}

Numbers in parentheses are three times the standard errors $(3 \sigma)$ referring to the last significant digit. a The present study. b Ref. [2]. c Ref. [3]. d See Fig. 3 for the definitions of the conformers. e $\mathrm{N} \cdots \mathrm{O}_{\text {carbonyl }}$ distances. ${ }^{\mathrm{f}}$ Assumed. g Dependent parameters. 


\section{Figure captions}

Fig. 1. Chemical structures of cotinine and nicotine.

Fig. 2. Experimental (dots) and theoretical (solid curve) molecular scattering intensities of cotinine; $\Delta s M(s)=s M(s)^{\text {obs }}-s M(s)^{\text {calc }}$. The theoretical curves were calculated from the best fitting parameters.

Fig. 3. Molecular models and atom numbering for the four possible conformers of cotinine. The abbreviations of the conformer, such as (ax, sc) represent the positions of the pyridine ring and the $\mathrm{H}_{18}-\mathrm{C}_{4}-\mathrm{C}_{7}-\mathrm{C}_{8}$ dihedral angle, respectively.

Fig. 4. The ring puckering angle $\alpha$ of cotinine and nicotine. The angle $\alpha$ of cotinine is defined as the dihedral angle between the $\mathrm{C}_{4} \mathrm{C}_{3} \mathrm{C}_{5}$ and $\mathrm{C}_{4} \mathrm{C}_{6} \mathrm{C}_{5}$ planes. The line $\mathrm{a}-\mathrm{b}$ is on the $\mathrm{C}_{4} \mathrm{C}_{3} \mathrm{C}_{5}$ plane and perpendicular to the $\mathrm{C}_{4}-\mathrm{C}_{5}$ bond. For nicotine, $\alpha$ is the dihedral angle between the $\mathrm{C}_{3} \mathrm{C}_{5} \mathrm{C}_{6} \mathrm{C}_{4}$ and $\mathrm{C}_{3} \mathrm{~N}_{1} \mathrm{C}_{4}$ planes.

Fig. 5. Experimental radial distribution curve of cotinine; $\Delta f(r)=f(r)^{\mathrm{obs}}-f(r)^{\text {calc }}$. The conformational composition of (ax, sc), (ax, ap), (eq, sp) and (eq, ap) used was $34 \%, 21 \%, 28 \%$ and $17 \%$, respectively. Distance distributions are indicated by vertical bars. Those for nonbonded $\mathrm{C} \cdots \mathrm{H}, \mathrm{N} \cdots \mathrm{H}$ and $\mathrm{H} \cdots \mathrm{H}$ pairs are not shown for simplicity. 
Fig. 1
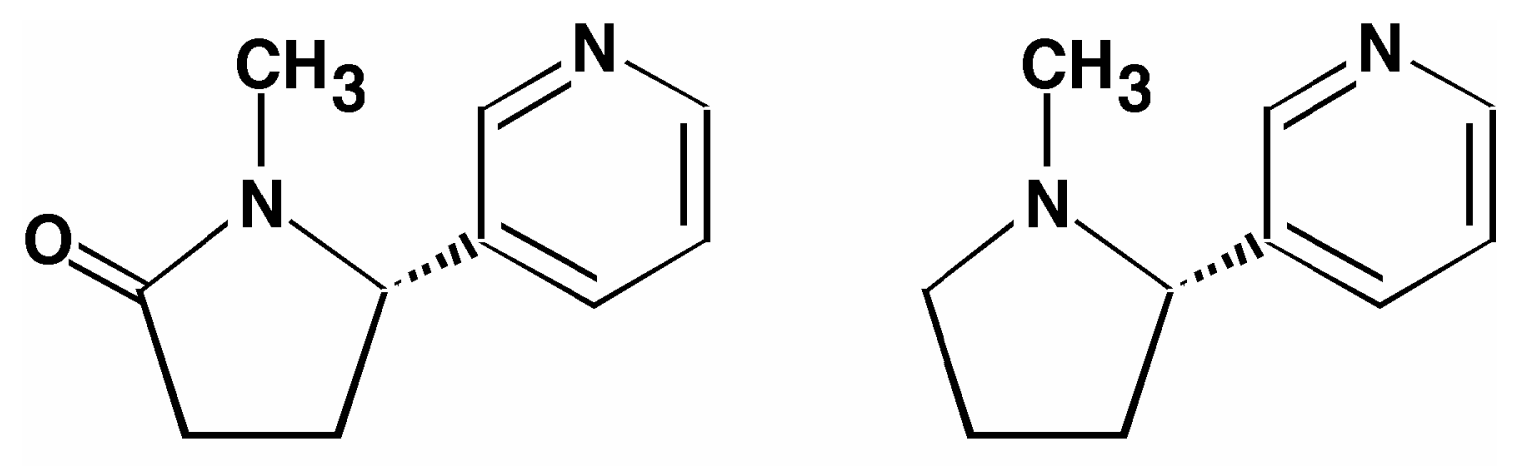

Cotinine

Nicotine 
Fig. 2

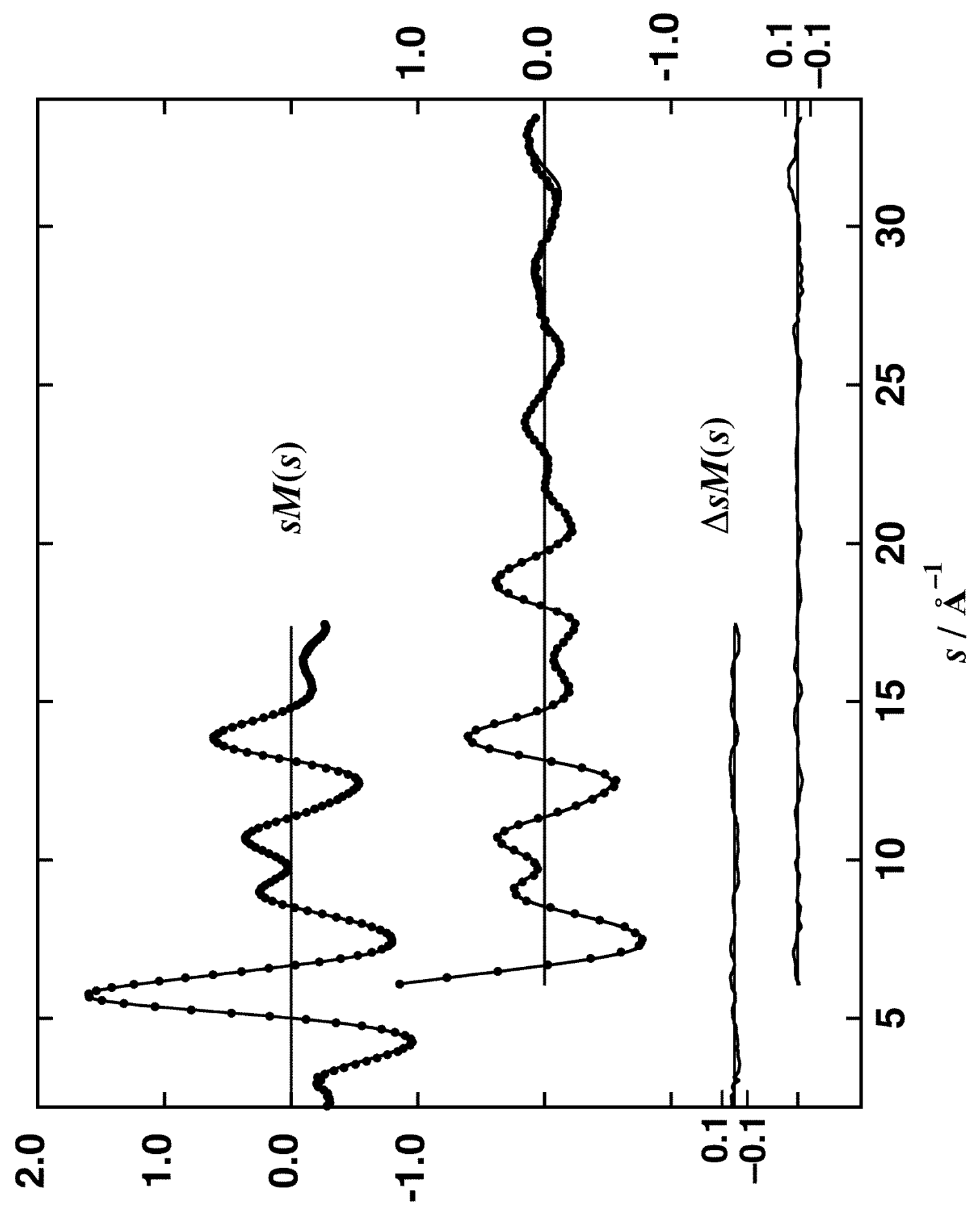


Fig. 3
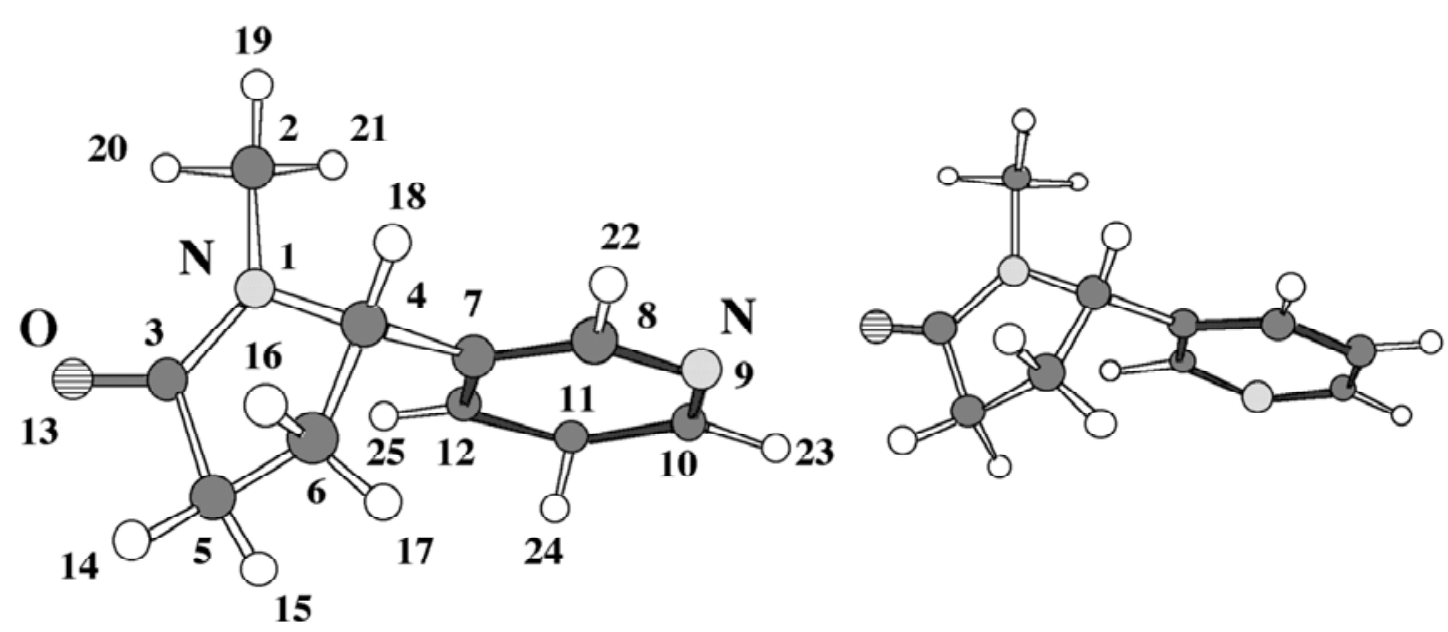

$$
(\mathrm{ax}, s c)
$$

$(\mathrm{ax}, a p)$

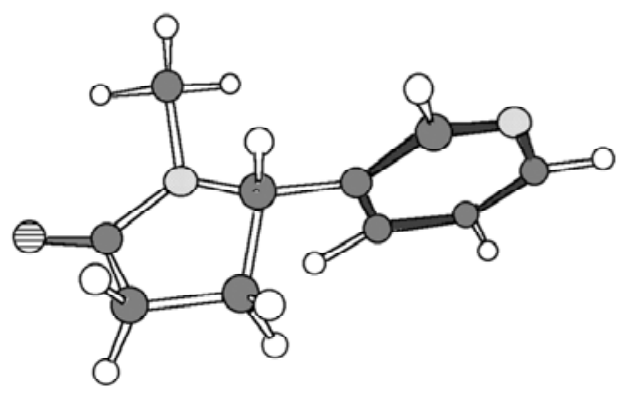

$(\mathrm{eq}, s p)$

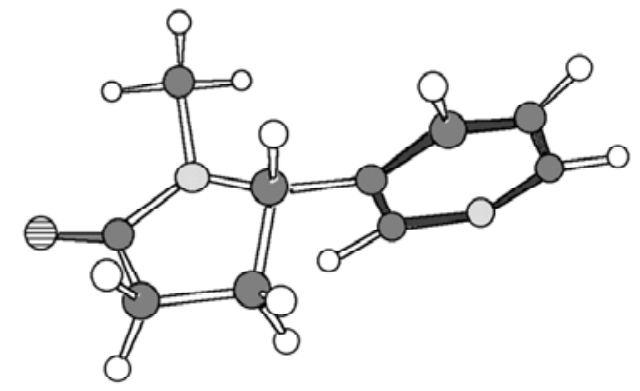

$(\mathrm{eq}, a p)$ 
Fig. 4

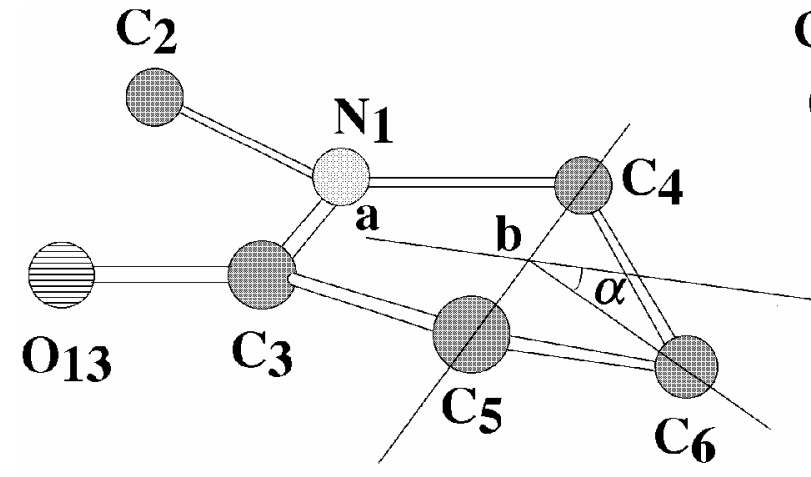

Cotinine
C2

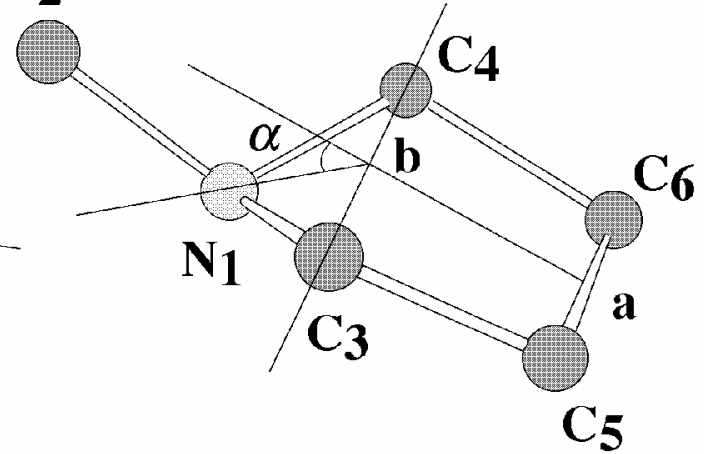

Nicotine 
Fig. 5

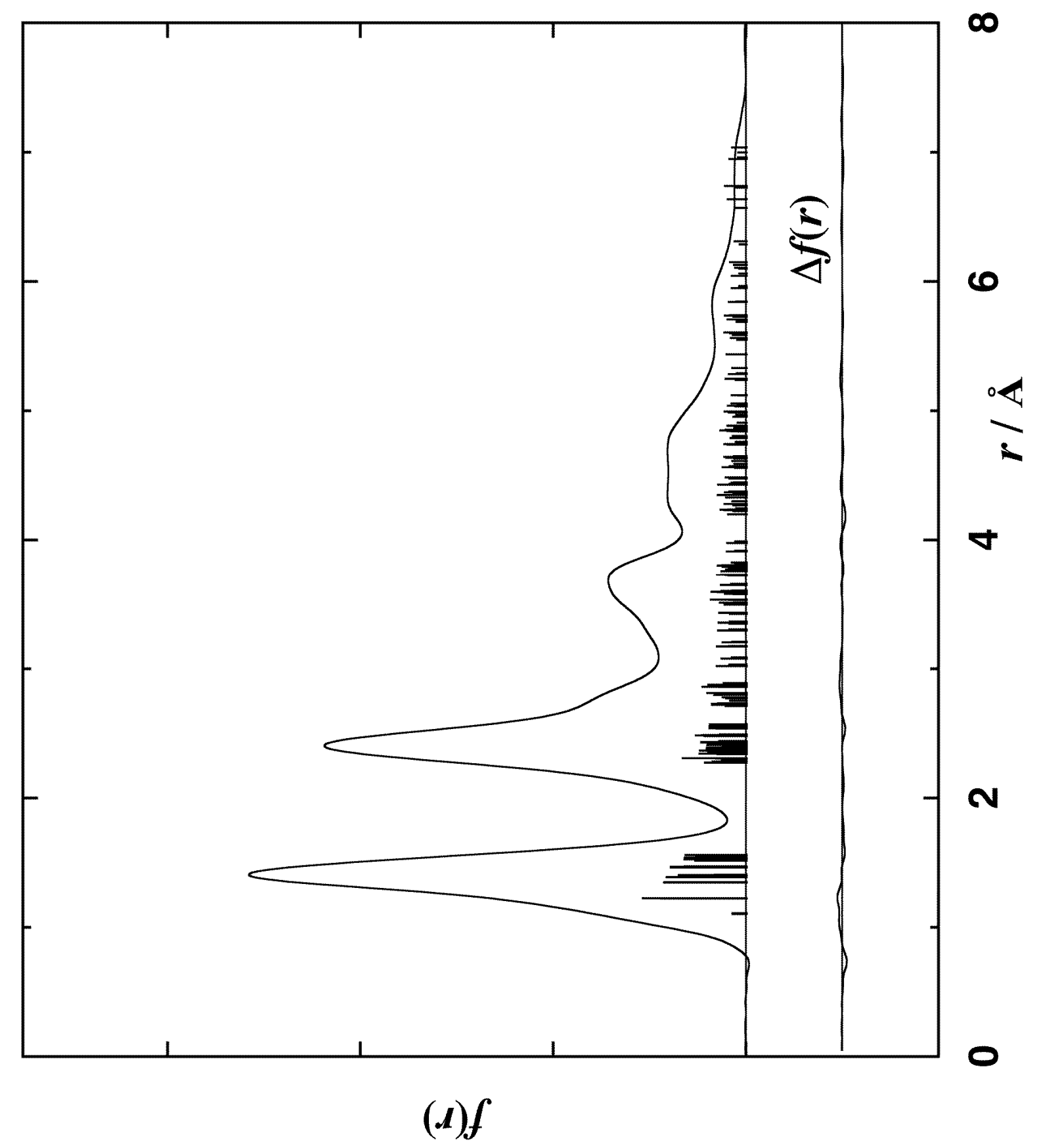




\section{Molecular structure of cotinine studied by gas electron diffraction combined with theoretical calculations}

\section{Tsuguhide Takeshima ${ }^{1}$, Hiroshi Takeuchi, Toru Egawa ${ }^{*}$ and Shigehiro Konaka}

Division of Chemistry, Graduate School of Science, Hokkaido University, Sapporo 060-0810, Japan

\section{Supplementary Material}

Table S1 The leveled total intensities $\left(I_{\mathrm{T}}\right)$ and the backgrounds $\left(I_{\mathrm{B}}\right)$ for cotinine.

Table S2 Definitions of internal coordinates of cotinine.

Table S3 The scaled harmonic force constants of the (eq, $s p$ ) conformer of cotinine.

Table S4 $r_{\mathrm{a}}$ Distances and mean amplitudes of the (ax, $s c$ ) conformer of cotinine ( $\AA$ ).

Table S5 The correlation matrix for cotinine.

Present address: Hokkaido Laboratory, Nissei Bio Co. Ltd., Eniwa 061-1374, Japan.

* Corresponding author. Tel.: +81-11-706-3506; fax: +81-11-706-4924.

E-mail address: egawa@sci.hokudai.ac.jp (T. Egawa). 
Table S1: The leveled total intensities $\left(I_{\mathrm{T}}\right)$ and the backgrounds $\left(I_{\mathrm{B}}\right)$ for cotinine a

Short camera distance

\begin{tabular}{|c|c|c|c|c|c|c|c|c|}
\hline$S$ & $I_{\mathrm{T}}$ & $I_{\mathrm{B}}$ & $S$ & $I_{\mathrm{T}}$ & $I_{\mathrm{B}}$ & $S$ & $I_{\mathrm{T}}$ & $I_{\mathrm{B}}$ \\
\hline 6.081 & 1.319 & 1.111 & 15.684 & 0.983 & 0.993 & 24.964 & 1.015 & 1.016 \\
\hline 6.283 & 1.241 & 1.106 & 15.881 & 0.984 & 0.992 & 25.153 & 1.016 & 1.017 \\
\hline 6.485 & 1.159 & 1.097 & 16.078 & 0.986 & 0.992 & 25.341 & 1.016 & 1.019 \\
\hline 6.687 & 1.082 & 1.086 & 16.275 & 0.987 & 0.991 & 25.529 & 1.017 & 1.020 \\
\hline 6.889 & 1.020 & 1.077 & 16.472 & 0.986 & 0.991 & 25.717 & 1.017 & 1.022 \\
\hline 7.091 & 0.978 & 1.069 & 16.668 & 0.984 & 0.990 & 25.905 & 1.018 & 1.023 \\
\hline 7.293 & 0.955 & 1.063 & 16.865 & 0.981 & 0.990 & 26.093 & 1.020 & 1.025 \\
\hline 7.494 & 0.950 & 1.059 & 17.061 & 0.979 & 0.990 & 26.280 & 1.022 & 1.026 \\
\hline 7.696 & 0.957 & 1.055 & 17.258 & 0.976 & 0.990 & 26.468 & 1.025 & 1.028 \\
\hline 7.897 & 0.967 & 1.051 & 17.454 & 0.976 & 0.989 & 26.655 & 1.028 & 1.029 \\
\hline 8.099 & 0.992 & 1.048 & 17.650 & 0.978 & 0.989 & 26.841 & 1.031 & 1.031 \\
\hline 8.300 & 1.015 & 1.045 & 17.845 & 0.984 & 0.989 & 27.028 & 1.033 & 1.033 \\
\hline 8.502 & 1.036 & 1.042 & 18.041 & 0.991 & 0.989 & 27.214 & 1.036 & 1.034 \\
\hline 8.703 & 1.056 & 1.039 & 18.236 & 0.998 & 0.989 & 27.400 & 1.037 & 1.036 \\
\hline 8.904 & 1.063 & 1.037 & 18.432 & 1.005 & 0.989 & 27.586 & 1.039 & 1.038 \\
\hline 9.105 & 1.062 & 1.035 & 18.627 & 1.009 & 0.990 & 27.772 & 1.041 & 1.040 \\
\hline 9.306 & 1.052 & 1.033 & 18.822 & 1.010 & 0.990 & 27.957 & 1.042 & 1.041 \\
\hline 9.507 & 1.041 & 1.031 & 19.016 & 1.008 & 0.990 & 28.142 & 1.045 & 1.043 \\
\hline 9.708 & 1.035 & 1.030 & 19.211 & 1.005 & 0.990 & 28.327 & 1.047 & 1.045 \\
\hline 9.909 & 1.036 & 1.028 & 19.405 & 1.000 & 0.991 & 28.512 & 1.050 & 1.047 \\
\hline 10.109 & 1.041 & 1.026 & 19.600 & 0.994 & 0.991 & 28.697 & 1.051 & 1.049 \\
\hline 10.310 & 1.049 & 1.025 & 19.794 & 0.989 & 0.991 & 28.881 & 1.053 & 1.051 \\
\hline 10.511 & 1.056 & 1.023 & 19.988 & 0.986 & 0.992 & 29.065 & 1.054 & 1.053 \\
\hline 10.711 & 1.057 & 1.022 & 20.181 & 0.983 & 0.992 & 29.249 & 1.056 & 1.055 \\
\hline 10.911 & 1.050 & 1.020 & 20.375 & 0.982 & 0.993 & 29.432 & 1.057 & 1.057 \\
\hline 11.111 & 1.038 & 1.019 & 20.568 & 0.983 & 0.993 & 29.616 & 1.058 & 1.059 \\
\hline 11.311 & 1.022 & 1.017 & 20.762 & 0.985 & 0.994 & 29.799 & 1.059 & 1.061 \\
\hline 11.511 & 1.007 & 1.016 & 20.955 & 0.987 & 0.994 & 29.981 & 1.061 & 1.063 \\
\hline 11.711 & 0.993 & 1.015 & 21.147 & 0.990 & 0.995 & 30.164 & 1.063 & 1.065 \\
\hline 11.911 & 0.981 & 1.013 & 21.340 & 0.993 & 0.996 & 30.346 & 1.064 & 1.067 \\
\hline 12.111 & 0.972 & 1.012 & 21.533 & 0.995 & 0.996 & 30.529 & 1.067 & 1.069 \\
\hline 12.310 & 0.965 & 1.010 & 21.725 & 0.997 & 0.997 & 30.710 & 1.068 & 1.072 \\
\hline 12.510 & 0.964 & 1.009 & 21.917 & 0.998 & 0.998 & 30.892 & 1.071 & 1.074 \\
\hline 12.709 & 0.970 & 1.008 & 22.109 & 0.998 & 0.999 & 31.074 & 1.073 & 1.076 \\
\hline 12.908 & 0.983 & 1.006 & 22.301 & 0.998 & 1.000 & 31.255 & 1.077 & 1.078 \\
\hline 13.107 & 1.001 & 1.005 & 22.492 & 0.999 & 1.001 & 31.436 & 1.080 & 1.081 \\
\hline 13.306 & 1.019 & 1.004 & 22.683 & 1.000 & 1.001 & 31.616 & 1.084 & 1.083 \\
\hline 13.505 & 1.035 & 1.003 & 22.875 & 1.003 & 1.002 & 31.797 & 1.087 & 1.086 \\
\hline 13.704 & 1.043 & 1.002 & 23.066 & 1.005 & 1.003 & 31.977 & 1.090 & 1.088 \\
\hline 13.902 & 1.044 & 1.000 & 23.256 & 1.008 & 1.005 & 32.157 & 1.093 & 1.090 \\
\hline 14.101 & 1.038 & 0.999 & 23.447 & 1.011 & 1.006 & 32.336 & 1.097 & 1.093 \\
\hline 14.299 & 1.026 & 0.998 & 23.637 & 1.013 & 1.007 & 32.516 & 1.099 & 1.096 \\
\hline 14.497 & 1.012 & 0.997 & 23.827 & 1.015 & 1.008 & 32.695 & 1.102 & 1.098 \\
\hline 14.695 & 1.000 & 0.996 & 24.017 & 1.015 & 1.009 & 32.874 & 1.105 & 1.101 \\
\hline 14.893 & 0.991 & 0.996 & 24.207 & 1.015 & 1.010 & 33.053 & 1.108 & 1.104 \\
\hline 15.091 & 0.985 & 0.995 & 24.397 & 1.015 & 1.012 & 33.231 & 1.110 & 1.107 \\
\hline 15.289 & 0.981 & 0.994 & 24.586 & 1.015 & 1.013 & 33.409 & 1.112 & 1.110 \\
\hline 15.486 & 0.981 & 0.993 & 24.775 & 1.015 & 1.014 & & & \\
\hline
\end{tabular}


Long camera distance

\begin{tabular}{|c|c|c|c|c|c|c|c|c|}
\hline$S$ & $I_{\mathrm{T}}$ & $I_{\mathrm{B}}$ & $S$ & $I_{\mathrm{T}}$ & $I_{\mathrm{B}}$ & $S$ & $I_{\mathrm{T}}$ & $I_{\mathrm{B}}$ \\
\hline 2.230 & 0.759 & 0.869 & 7.386 & 0.912 & 1.022 & 12.497 & 0.938 & 0.980 \\
\hline 2.332 & 0.760 & 0.874 & 7.487 & 0.912 & 1.021 & 12.597 & 0.940 & 0.980 \\
\hline 2.433 & 0.771 & 0.880 & 7.588 & 0.914 & 1.019 & 12.696 & 0.944 & 0.979 \\
\hline 2.534 & 0.787 & 0.887 & 7.689 & 0.918 & 1.018 & 12.796 & 0.950 & 0.978 \\
\hline 2.636 & 0.797 & 0.895 & 7.789 & 0.925 & 1.017 & 12.895 & 0.957 & 0.978 \\
\hline 2.737 & 0.819 & 0.904 & 7.890 & 0.934 & 1.016 & 12.995 & 0.965 & 0.977 \\
\hline 2.838 & 0.844 & 0.913 & 7.991 & 0.944 & 1.015 & 13.094 & 0.973 & 0.977 \\
\hline 2.940 & 0.859 & 0.922 & 8.091 & 0.956 & 1.013 & 13.194 & 0.983 & 0.976 \\
\hline 3.041 & 0.859 & 0.931 & 8.192 & 0.968 & 1.012 & 13.293 & 0.992 & 0.975 \\
\hline 3.142 & 0.878 & 0.941 & 8.293 & 0.982 & 1.011 & 13.392 & 1.001 & 0.975 \\
\hline 3.243 & 0.872 & 0.950 & 8.393 & 0.995 & 1.010 & 13.492 & 1.008 & 0.974 \\
\hline 3.345 & 0.862 & 0.959 & 8.494 & 1.006 & 1.009 & 13.591 & 1.013 & 0.974 \\
\hline 3.446 & 0.851 & 0.968 & 8.594 & 1.016 & 1.008 & 13.690 & 1.015 & 0.973 \\
\hline 3.547 & 0.836 & 0.976 & 8.695 & 1.025 & 1.007 & 13.789 & 1.017 & 0.973 \\
\hline 3.648 & 0.823 & 0.984 & 8.795 & 1.030 & 1.007 & 13.888 & 1.016 & 0.972 \\
\hline 3.750 & 0.812 & 0.991 & 8.896 & 1.033 & 1.006 & 13.988 & 1.013 & 0.972 \\
\hline 3.851 & 0.801 & 0.998 & 8.996 & 1.033 & 1.005 & 14.087 & 1.009 & 0.971 \\
\hline 3.952 & 0.791 & 1.005 & 9.097 & 1.030 & 1.004 & 14.186 & 1.003 & 0.971 \\
\hline 4.053 & 0.790 & 1.010 & 9.197 & 1.026 & 1.003 & 14.285 & 0.998 & 0.970 \\
\hline 4.155 & 0.786 & 1.016 & 9.297 & 1.020 & 1.002 & 14.384 & 0.991 & 0.970 \\
\hline 4.256 & 0.791 & 1.021 & 9.398 & 1.015 & 1.002 & 14.483 & 0.984 & 0.970 \\
\hline 4.357 & 0.803 & 1.025 & 9.498 & 1.009 & 1.001 & 14.582 & 0.979 & 0.969 \\
\hline 4.458 & 0.821 & 1.029 & 9.599 & 1.005 & 1.000 & 14.681 & 0.973 & 0.969 \\
\hline 4.559 & 0.846 & 1.032 & 9.699 & 1.002 & 0.999 & 14.779 & 0.969 & 0.969 \\
\hline 4.660 & 0.876 & 1.035 & 9.799 & 1.002 & 0.999 & 14.878 & 0.965 & 0.968 \\
\hline 4.762 & 0.915 & 1.037 & 9.899 & 1.002 & 0.998 & 14.977 & 0.962 & 0.968 \\
\hline 4.863 & 0.964 & 1.039 & 10.000 & 1.005 & 0.997 & 15.076 & 0.960 & 0.968 \\
\hline 4.964 & 1.016 & 1.041 & 10.100 & 1.008 & 0.997 & 15.175 & 0.959 & 0.968 \\
\hline 5.065 & 1.077 & 1.042 & 10.200 & 1.013 & 0.996 & 15.273 & 0.958 & 0.968 \\
\hline 5.166 & 1.138 & 1.043 & 10.300 & 1.017 & 0.995 & 15.372 & 0.957 & 0.968 \\
\hline 5.267 & 1.200 & 1.044 & 10.400 & 1.021 & 0.994 & 15.471 & 0.958 & 0.968 \\
\hline 5.368 & 1.253 & 1.044 & 10.500 & 1.024 & 0.994 & 15.569 & 0.958 & 0.968 \\
\hline 5.469 & 1.296 & 1.044 & 10.601 & 1.026 & 0.993 & 15.668 & 0.959 & 0.969 \\
\hline 5.570 & 1.324 & 1.044 & 10.701 & 1.026 & 0.992 & 15.766 & 0.960 & 0.969 \\
\hline 5.671 & 1.336 & 1.043 & 10.801 & 1.023 & 0.991 & 15.865 & 0.962 & 0.970 \\
\hline 5.772 & 1.332 & 1.043 & 10.901 & 1.019 & 0.991 & 15.963 & 0.963 & 0.970 \\
\hline 5.873 & 1.315 & 1.042 & 11.001 & 1.013 & 0.990 & 16.062 & 0.964 & 0.971 \\
\hline 5.974 & 1.288 & 1.041 & 11.101 & 1.006 & 0.989 & 16.160 & 0.965 & 0.971 \\
\hline 6.075 & 1.253 & 1.040 & 11.201 & 0.999 & 0.989 & 16.258 & 0.966 & 0.972 \\
\hline 6.176 & 1.214 & 1.039 & 11.301 & 0.991 & 0.988 & 16.357 & 0.967 & 0.973 \\
\hline 6.277 & 1.176 & 1.038 & 11.400 & 0.984 & 0.987 & 16.455 & 0.967 & 0.974 \\
\hline 6.378 & 1.137 & 1.036 & 11.500 & 0.976 & 0.987 & 16.553 & 0.967 & 0.974 \\
\hline 6.479 & 1.097 & 1.035 & 11.600 & 0.970 & 0.986 & 16.651 & 0.967 & 0.975 \\
\hline 6.580 & 1.061 & 1.033 & 11.700 & 0.965 & 0.985 & 16.749 & 0.966 & 0.976 \\
\hline 6.681 & 1.027 & 1.032 & 11.800 & 0.959 & 0.985 & 16.847 & 0.966 & 0.976 \\
\hline 6.782 & 0.997 & 1.031 & 11.899 & 0.954 & 0.984 & 16.946 & 0.965 & 0.977 \\
\hline 6.882 & 0.971 & 1.029 & 11.999 & 0.950 & 0.983 & 17.044 & 0.964 & 0.978 \\
\hline 6.983 & 0.950 & 1.028 & 12.099 & 0.946 & 0.983 & 17.142 & 0.963 & 0.978 \\
\hline 7.084 & 0.934 & 1.026 & 12.199 & 0.942 & 0.982 & 17.240 & 0.963 & 0.979 \\
\hline 7.185 & 0.923 & 1.025 & 12.298 & 0.940 & 0.982 & 17.337 & 0.964 & 0.980 \\
\hline 7.286 & 0.916 & 1.023 & 12.398 & 0.938 & 0.981 & 17.435 & 0.965 & 0.980 \\
\hline
\end{tabular}

a The $s$-values $(s=(4 \pi / \lambda) \sin (\theta / 2))$ are in $\AA^{-1}$ units. 
Table S2: Definitions of internal coordinates of cotinine

\begin{tabular}{|c|c|}
\hline Coordinates & Definitions a \\
\hline$s_{1}$ & $r(\mathrm{~N} 1-\mathrm{C} 2)$ \\
\hline$s_{2}$ & $r(\mathrm{~N} 1-\mathrm{C} 3)$ \\
\hline$s_{3}$ & $r(\mathrm{~N} 1-\mathrm{C} 4)$ \\
\hline$s_{4}$ & $r(\mathrm{C} 3-\mathrm{C} 5)$ \\
\hline$S_{5}$ & $r(\mathrm{C} 4-\mathrm{C} 6)$ \\
\hline$s_{6}$ & $r(\mathrm{C} 5-\mathrm{C} 6)$ \\
\hline$s_{7}$ & $r(\mathrm{C} 3=\mathrm{O} 13)$ \\
\hline$S_{8}$ & $r(\mathrm{C} 2-\mathrm{H} 19)+r(\mathrm{C} 2-\mathrm{H} 20)+r(\mathrm{C} 2-\mathrm{H} 21)$ \\
\hline$S_{9}$ & $2 r(\mathrm{C} 2-\mathrm{H} 19)-r(\mathrm{C} 2-\mathrm{H} 20)-r(\mathrm{C} 2-\mathrm{H} 21)$ \\
\hline$s_{10}$ & $r(\mathrm{C} 2-\mathrm{H} 20)-r(\mathrm{C} 2-\mathrm{H} 21)$ \\
\hline$s_{11}$ & $r(\mathrm{C} 5-\mathrm{H} 14)+r(\mathrm{C} 5-\mathrm{H} 15)$ \\
\hline$s_{12}$ & $r(\mathrm{C} 5-\mathrm{H} 14)-r(\mathrm{C} 5-\mathrm{H} 15)$ \\
\hline$s_{13}$ & $r(\mathrm{C} 6-\mathrm{H} 16)+r(\mathrm{C} 6-\mathrm{H} 17)$ \\
\hline$s_{14}$ & $r(\mathrm{C} 6-\mathrm{H} 16)-r(\mathrm{C} 6-\mathrm{H} 17)$ \\
\hline$s_{15}$ & $r(\mathrm{C} 4-\mathrm{C} 7)$ \\
\hline$s_{16}$ & $r(\mathrm{C} 4-\mathrm{H} 18)$ \\
\hline$s_{17}$ & $r(\mathrm{C} 8-\mathrm{N} 9)+r(\mathrm{~N} 9-\mathrm{C} 10)$ \\
\hline$s_{18}$ & $r(\mathrm{C} 8-\mathrm{N} 9)-r(\mathrm{~N} 9-\mathrm{C} 10)$ \\
\hline$s_{19}$ & $r(\mathrm{C} 7-\mathrm{C} 8)+r(\mathrm{C} 10-\mathrm{C} 11)$ \\
\hline$s_{20}$ & $r(\mathrm{C} 7-\mathrm{C} 8)-r(\mathrm{C} 10-\mathrm{C} 11)$ \\
\hline$s_{21}$ & $r(\mathrm{C} 7-\mathrm{C} 12)+r(\mathrm{C} 11-\mathrm{C} 12)$ \\
\hline$s_{22}$ & $r(\mathrm{C} 7-\mathrm{C} 12)-r(\mathrm{C} 11-\mathrm{C} 12)$ \\
\hline$s_{23}$ & $r(\mathrm{C} 8-\mathrm{H} 22)+r(\mathrm{C} 10-\mathrm{H} 23)$ \\
\hline$s_{24}$ & $r(\mathrm{C} 8-\mathrm{H} 22)-r(\mathrm{C} 10-\mathrm{H} 23)$ \\
\hline$s_{25}$ & $r(\mathrm{C} 11-\mathrm{H} 24)$ \\
\hline$s_{26}$ & $r(\mathrm{C} 12-\mathrm{H} 25)$ \\
\hline$s_{27}$ & $\angle(\mathrm{C} 2-\mathrm{N} 1-\mathrm{C} 3)-\angle(\mathrm{C} 2-\mathrm{N} 1-\mathrm{C} 4)$ \\
\hline$s_{28}$ & $\angle(\mathrm{N} 1-\mathrm{C} 3=\mathrm{O} 13)-\angle(\mathrm{C} 5-\mathrm{C} 3=\mathrm{O} 13)$ \\
\hline$s_{29}$ & $\begin{array}{l}\angle(\mathrm{C} 3-\mathrm{C} 5-\mathrm{H} 14)+\angle(\mathrm{C} 3-\mathrm{C} 5-\mathrm{H} 15)-\angle(\mathrm{C} 6-\mathrm{C} 5-\mathrm{H} 14)-\angle(\mathrm{C} 6-\mathrm{C} 5- \\
\mathrm{H} 15)\end{array}$ \\
\hline
\end{tabular}


Table S2 continued

\begin{tabular}{|c|c|}
\hline$s_{30}$ & $\begin{array}{l}\angle(\mathrm{C} 3-\mathrm{C} 5-\mathrm{H} 14)-\angle(\mathrm{C} 3-\mathrm{C} 5-\mathrm{H} 15)-\angle(\mathrm{C} 6-\mathrm{C} 5-\mathrm{H} 14)+\angle(\mathrm{C} 6-\mathrm{C} 5- \\
\mathrm{H} 15)\end{array}$ \\
\hline$s_{31}$ & $\begin{array}{l}\angle(\mathrm{C} 3-\mathrm{C} 5-\mathrm{H} 14)-\angle(\mathrm{C} 3-\mathrm{C} 5-\mathrm{H} 15)+\angle(\mathrm{C} 6-\mathrm{C} 5-\mathrm{H} 14)-\angle(\mathrm{C} 6-\mathrm{C} 5- \\
\mathrm{H} 15)\end{array}$ \\
\hline$s_{32}$ & $\begin{array}{l}-\angle(\mathrm{C} 3-\mathrm{C} 5-\mathrm{H} 14)-\angle(\mathrm{C} 3-\mathrm{C} 5-\mathrm{H} 15)-\angle(\mathrm{C} 6-\mathrm{C} 5-\mathrm{H} 14)-\angle(\mathrm{C} 6-\mathrm{C} 5 \\
-\mathrm{H} 15)+4 \angle(\mathrm{H} 14-\mathrm{C} 5-\mathrm{H} 15)\end{array}$ \\
\hline$s_{33}$ & $\begin{array}{l}\angle(\mathrm{C} 5-\mathrm{C} 6-\mathrm{H} 16)+\angle(\mathrm{C} 5-\mathrm{C} 6-\mathrm{H} 17)-\angle(\mathrm{C} 4-\mathrm{C} 6-\mathrm{H} 16)-\angle(\mathrm{C} 4-\mathrm{C} 6- \\
\mathrm{H} 17)\end{array}$ \\
\hline$s_{34}$ & $\begin{array}{l}\angle(\mathrm{C} 5-\mathrm{C} 6-\mathrm{H} 16)-\angle(\mathrm{C} 5-\mathrm{C} 6-\mathrm{H} 17)-\angle(\mathrm{C} 4-\mathrm{C} 6-\mathrm{H} 16)+ \\
\angle(\mathrm{C} 4-\mathrm{C} 6-\mathrm{H} 17)\end{array}$ \\
\hline$s_{35}$ & $\begin{array}{l}\angle(\mathrm{C} 5-\mathrm{C} 6-\mathrm{H} 16)-\angle(\mathrm{C} 5-\mathrm{C} 6-\mathrm{H} 17)+\angle(\mathrm{C} 4-\mathrm{C} 6-\mathrm{H} 16)- \\
\angle(\mathrm{C} 4-\mathrm{C} 6-\mathrm{H} 17)\end{array}$ \\
\hline$s_{36}$ & $\begin{array}{l}-\angle(\mathrm{C} 5-\mathrm{C} 6-\mathrm{H} 16)-\angle(\mathrm{C} 5-\mathrm{C} 6-\mathrm{H} 17)-\angle(\mathrm{C} 4-\mathrm{C} 6-\mathrm{H} 16)- \\
\angle(\mathrm{C} 4-\mathrm{C} 6-\mathrm{H} 17)+4 \angle(\mathrm{H} 16-\mathrm{C} 6-\mathrm{H} 17)\end{array}$ \\
\hline$s_{37}$ & $2 \angle(\mathrm{C} 7-\mathrm{C} 4-\mathrm{H} 18)-\angle(\mathrm{C} 1-\mathrm{C} 4-\mathrm{H} 18)-\angle(\mathrm{C} 6-\mathrm{C} 4-\mathrm{H} 18)$ \\
\hline$s_{38}$ & $\angle(\mathrm{C} 1-\mathrm{C} 4-\mathrm{H} 18)-\angle(\mathrm{C} 6-\mathrm{C} 4-\mathrm{H} 18)$ \\
\hline$s_{39}$ & $2 \angle(\mathrm{C} 7-\mathrm{C} 4-\mathrm{N} 1)-\angle(\mathrm{C} 7-\mathrm{C} 4-\mathrm{C} 6)-\angle(\mathrm{C} 7-\mathrm{C} 4-\mathrm{H} 18)$ \\
\hline$s_{40}$ & $\angle(\mathrm{C} 7-\mathrm{C} 4-\mathrm{C} 6)-\angle(\mathrm{C} 7-\mathrm{C} 4-\mathrm{H} 18)$ \\
\hline$s_{41}$ & $\begin{array}{l}\angle(\mathrm{N} 1-\mathrm{C} 2-\mathrm{H} 19)+\angle(\mathrm{N} 1-\mathrm{C} 2-\mathrm{H} 20)+\angle(\mathrm{N} 1-\mathrm{C} 2-\mathrm{H} 21)- \\
\angle(\mathrm{H} 19-\mathrm{C} 2-\mathrm{H} 20)-\angle(\mathrm{H} 19-\mathrm{C} 2-\mathrm{H} 21)-\angle(\mathrm{H} 20-\mathrm{C} 2-\mathrm{H} 21)\end{array}$ \\
\hline$s_{42}$ & $2 \angle(\mathrm{N} 1-\mathrm{C} 2-\mathrm{H} 19)-\angle(\mathrm{N} 1-\mathrm{C} 2-\mathrm{H} 20)-\angle(\mathrm{N} 1-\mathrm{C} 2-\mathrm{H} 21)$ \\
\hline$s_{43}$ & $\angle(\mathrm{N} 1-\mathrm{C} 2-\mathrm{H} 20)-\angle(\mathrm{N} 1-\mathrm{C} 2-\mathrm{H} 21)$ \\
\hline$s_{44}$ & $-\angle(\mathrm{H} 19-\mathrm{C} 2-\mathrm{H} 20)-\angle(\mathrm{H} 19-\mathrm{C} 2-\mathrm{H} 21)+2 \angle(\mathrm{H} 20-\mathrm{C} 2-\mathrm{H} 21)$ \\
\hline$s_{45}$ & $\angle(\mathrm{H} 19-\mathrm{C} 2-\mathrm{H} 20)-\angle(\mathrm{H} 19-\mathrm{C} 2-\mathrm{H} 21)$ \\
\hline$s_{46}$ & $\begin{array}{l}4 \angle(\mathrm{C} 3-\mathrm{N} 1-\mathrm{C} 4)-2 \angle(\mathrm{C} 2-\mathrm{N} 1-\mathrm{C} 3)-2 \angle(\mathrm{C} 2-\mathrm{N} 1-\mathrm{C} 4)-4 \angle(\mathrm{N} 1-\mathrm{C} 3 \\
-\mathrm{C} 5)+2 \angle(\mathrm{N} 1-\mathrm{C} 3=\mathrm{O} 13)+2 \angle(\mathrm{C} 5-\mathrm{C} 3=\mathrm{O} 13)-10 \angle(\mathrm{N} 1-\mathrm{C} 4-\mathrm{C} 6)+ \\
2 \angle(\mathrm{C} 7-\mathrm{C} 4-\mathrm{C} 6)+2 \angle(\mathrm{C} 7-\mathrm{C} 4-\mathrm{N} 1)+2 \angle(\mathrm{N} 1-\mathrm{C} 4-\mathrm{H} 18)+2 \angle(\mathrm{C} 6-\mathrm{C} \\
4-\mathrm{H} 18)+2 \angle(\mathrm{C} 7-\mathrm{C} 4-\mathrm{H} 18)+5 \angle(\mathrm{C} 3-\mathrm{C} 5-\mathrm{C} 6)-\angle(\mathrm{C} 3-\mathrm{C} 5-\mathrm{H} 14)- \\
\angle(\mathrm{C} 3-\mathrm{C} 5-\mathrm{H} 15)-\angle(\mathrm{C} 6-\mathrm{C} 5-\mathrm{H} 14)-\angle(\mathrm{C} 6-\mathrm{C} 5-\mathrm{H} 15)-\angle(\mathrm{H} 14-\mathrm{C} 5 \\
-\mathrm{H} 15)+5 \angle(\mathrm{C} 5-\mathrm{C} 6-\mathrm{C} 4)-\angle(\mathrm{C} 5-\mathrm{C} 6-\mathrm{H} 16)-\angle(\mathrm{C} 5-\mathrm{C} 6-\mathrm{H} 17)- \\
\angle(\mathrm{C} 4-\mathrm{C} 6-\mathrm{H} 16)-\angle(\mathrm{C} 4-\mathrm{C} 6-\mathrm{H} 17)-\angle(\mathrm{C} 16-\mathrm{C} 6-\mathrm{H} 17)\end{array}$ \\
\hline
\end{tabular}


Table S2 continued

\begin{tabular}{|c|c|}
\hline \multirow[t]{6}{*}{$s_{47}$} & $2 \angle(\mathrm{N} 1-\mathrm{C} 3-\mathrm{C} 5)-\angle(\mathrm{N} 1-\mathrm{C} 3=\mathrm{O} 13)-\angle(\mathrm{C} 5-\mathrm{C} 3=\mathrm{O} 13)-5 \angle(\mathrm{N} 1-\mathrm{C} 4$ \\
\hline & $-\mathrm{C} 6)+\angle(\mathrm{C} 7-\mathrm{C} 4-\mathrm{C} 6)+\angle(\mathrm{C} 7-\mathrm{C} 4-\mathrm{N} 1)+\angle(\mathrm{N} 1-\mathrm{C} 4-\mathrm{H} 18)+\angle(\mathrm{C} 6-$ \\
\hline & $\mathrm{C} 4-\mathrm{H} 18)+\angle(\mathrm{C} 7-\mathrm{C} 4-\mathrm{H} 18)-5 \angle(\mathrm{C} 3-\mathrm{C} 5-\mathrm{C} 6)+\angle(\mathrm{C} 3-\mathrm{C} 5-\mathrm{H} 14)+$ \\
\hline & $\angle(\mathrm{C} 3-\mathrm{C} 5-\mathrm{H} 15)+\angle(\mathrm{C} 6-\mathrm{C} 5-\mathrm{H} 14)+\angle(\mathrm{C} 6-\mathrm{C} 5-\mathrm{H} 15)+\angle(\mathrm{H} 14-\mathrm{C} 5$ \\
\hline & $-\mathrm{H} 15)+5 \angle(\mathrm{C} 5-\mathrm{C} 6-\mathrm{C} 4)-\angle(\mathrm{C} 5-\mathrm{C} 6-\mathrm{H} 16)-\angle(\mathrm{C} 5-\mathrm{C} 6-\mathrm{H} 17)-$ \\
\hline & $\angle(\mathrm{C} 4-\mathrm{C} 6-\mathrm{H} 16)-\angle(\mathrm{C} 4-\mathrm{C} 6-\mathrm{H} 17)-\angle(\mathrm{C} 16-\mathrm{C} 6-\mathrm{H} 17)$ \\
\hline \multirow[t]{2}{*}{$s_{48}$} & $\angle(\mathrm{N} 9-\mathrm{C} 8-\mathrm{H} 22)-\angle(\mathrm{C} 7-\mathrm{C} 8-\mathrm{H} 22)+\angle(\mathrm{N} 9-\mathrm{C} 10-\mathrm{H} 23)-$ \\
\hline & $\angle(\mathrm{C} 11-\mathrm{C} 10-\mathrm{H} 23)$ \\
\hline \multirow[t]{2}{*}{$s_{49}$} & $\angle(\mathrm{N} 9-\mathrm{C} 8-\mathrm{H} 22)-\angle(\mathrm{C} 7-\mathrm{C} 8-\mathrm{H} 22)-\angle(\mathrm{N} 9-\mathrm{C} 10-\mathrm{H} 23)+$ \\
\hline & $\angle(\mathrm{C} 11-\mathrm{C} 10-\mathrm{H} 23)$ \\
\hline$S_{50}$ & $\angle(\mathrm{C} 4-\mathrm{C} 7-\mathrm{C} 8)-\angle(\mathrm{C} 4-\mathrm{C} 7-\mathrm{C} 12)$ \\
\hline$s_{51}$ & $\angle(\mathrm{C} 10-\mathrm{C} 11-\mathrm{H} 24)-\angle(\mathrm{C} 12-\mathrm{C} 11-\mathrm{H} 24)$ \\
\hline$S_{52}$ & $\angle(\mathrm{C} 11-\mathrm{C} 12-\mathrm{H} 25)-\angle(\mathrm{C} 7-\mathrm{C} 12-\mathrm{H} 25)$ \\
\hline \multirow[t]{5}{*}{$s_{53}$} & $-2 \angle(\mathrm{C} 8-\mathrm{C} 7-\mathrm{C} 12)+\angle(\mathrm{C} 4-\mathrm{C} 7-\mathrm{C} 8)+\angle(\mathrm{C} 4-\mathrm{C} 7-\mathrm{C} 12)-2 \angle(\mathrm{C} 7-\mathrm{C}$ \\
\hline & $8-\mathrm{N} 9)+\angle(\mathrm{C} 7-\mathrm{C} 8-\mathrm{H} 22)+\angle(\mathrm{N} 9-\mathrm{C} 8-\mathrm{H} 22)+2 \angle(\mathrm{C} 8-\mathrm{N} 9-\mathrm{C} 10)-2$ \\
\hline & $\angle(\mathrm{N} 9-\mathrm{C} 10-\mathrm{C} 11)+\angle(\mathrm{N} 9-\mathrm{C} 10-\mathrm{H} 23)+\angle(\mathrm{C} 11-\mathrm{C} 10-\mathrm{H} 23)-2 \angle(\mathrm{C}$ \\
\hline & $10-\mathrm{C} 11-\mathrm{C} 12)+\angle(\mathrm{C} 10-\mathrm{C} 11-\mathrm{H} 24)+\angle(\mathrm{C} 12-\mathrm{C} 11-\mathrm{H} 24)+4 \angle(\mathrm{C} 7-$ \\
\hline & C12-C11)-2 $\angle(C 7-C 12-H 25)-2 \angle(C 11-C 12-H 25)$ \\
\hline \multirow[t]{5}{*}{$S_{54}$} & $2 \angle(\mathrm{C} 8-\mathrm{C} 7-\mathrm{C} 12)-\angle(\mathrm{C} 4-\mathrm{C} 7-\mathrm{C} 8)-\angle(\mathrm{C} 4-\mathrm{C} 7-\mathrm{C} 12)-2 \angle(\mathrm{C} 7-\mathrm{C} 8-$ \\
\hline & $\mathrm{N} 9)+\angle(\mathrm{C} 7-\mathrm{C} 8-\mathrm{H} 22)+\angle(\mathrm{N} 9-\mathrm{C} 8-\mathrm{H} 22)+2 \angle(\mathrm{C} 8-\mathrm{N} 9-\mathrm{C} 10)-$ \\
\hline & $2 \angle(\mathrm{N} 9-\mathrm{C} 10-\mathrm{C} 11)+\angle(\mathrm{N} 9-\mathrm{C} 10-\mathrm{H} 23)+\angle(\mathrm{C} 11-\mathrm{C} 10-\mathrm{H} 23)+$ \\
\hline & $2 \angle(\mathrm{C} 10-\mathrm{C} 11-\mathrm{C} 12)-\angle(\mathrm{C} 10-\mathrm{C} 11-\mathrm{H} 24)-\angle(\mathrm{C} 12-\mathrm{C} 11-\mathrm{H} 24)-$ \\
\hline & $4 \angle(\mathrm{C} 7-\mathrm{C} 12-\mathrm{C} 11)+2 \angle(\mathrm{C} 7-\mathrm{C} 12-\mathrm{H} 25)+2 \angle(\mathrm{C} 11-\mathrm{C} 12-\mathrm{H} 25)$ \\
\hline \multirow[t]{4}{*}{$S_{55}$} & $2 \angle(\mathrm{C} 8-\mathrm{C} 7-\mathrm{C} 12)-\angle(\mathrm{C} 4-\mathrm{C} 7-\mathrm{C} 8)-\angle(\mathrm{C} 4-\mathrm{C} 7-\mathrm{C} 12)-2 \angle(\mathrm{C} 7-\mathrm{C} 8-$ \\
\hline & $\mathrm{N} 9)+\angle(\mathrm{C} 7-\mathrm{C} 8-\mathrm{H} 22)+\angle(\mathrm{N} 9-\mathrm{C} 8-\mathrm{H} 22)+2 \angle(\mathrm{N} 9-\mathrm{C} 10-\mathrm{C} 11)-$ \\
\hline & $\angle(\mathrm{N} 9-\mathrm{C} 10-\mathrm{H} 23)-\angle(\mathrm{C} 11-\mathrm{C} 10-\mathrm{H} 23)-2 \angle(\mathrm{C} 10-\mathrm{C} 11-\mathrm{C} 12)+$ \\
\hline & $\angle(\mathrm{C} 10-\mathrm{C} 11-\mathrm{H} 24)+\angle(\mathrm{C} 12-\mathrm{C} 11-\mathrm{H} 24)$ \\
\hline$S_{56}$ & $\tau(\mathrm{N} 1-\mathrm{C} 2)$ \\
\hline$s_{57}$ & $\tau(\mathrm{N} 1-\mathrm{C} 3)-\tau(\mathrm{C} 3-\mathrm{C} 5)+\tau(\mathrm{C} 4-\mathrm{C} 6)-\tau(\mathrm{N} 1-\mathrm{C} 4)$ \\
\hline$s_{58}$ & $\tau(\mathrm{N} 1-\mathrm{C} 3)-2 \tau(\mathrm{C} 3-\mathrm{C} 5)+2 \tau(\mathrm{C} 5-\mathrm{C} 6)-2 \tau(\mathrm{C} 4-\mathrm{C} 6)+\tau(\mathrm{N} 1-\mathrm{C} 4)$ \\
\hline$S_{59}$ & $\tau(\mathrm{C} 4-\mathrm{C} 7)$ \\
\hline
\end{tabular}


Table S2 continued

\begin{tabular}{ll}
\hline$s_{60}$ & $-\tau(\mathrm{C} 8-\mathrm{N} 9)+2 \tau(\mathrm{C} 7-\mathrm{C} 8)-\tau(\mathrm{C} 7-\mathrm{C} 12)-\tau(\mathrm{C} 12-\mathrm{C} 11)$ \\
& $+2 \tau(\mathrm{C} 10-\mathrm{C} 11)-\tau(\mathrm{N} 9-\mathrm{C} 10)$ \\
$s_{61}$ & $\tau(\mathrm{C} 8-\mathrm{N} 9)-\tau(\mathrm{C} 7-\mathrm{C} 8)+\tau(\mathrm{C} 7-\mathrm{C} 12)-\tau(\mathrm{C} 12-\mathrm{C} 11)$ \\
& $+\tau(\mathrm{C} 10-\mathrm{C} 11)-\tau(\mathrm{N} 9-\mathrm{C} 10)$ \\
$s_{62}$ & $\tau(\mathrm{C} 8-\mathrm{N} 9)-\tau(\mathrm{C} 7-\mathrm{C} 12)+\tau(\mathrm{C} 12-\mathrm{C} 11)-\tau(\mathrm{N} 9-\mathrm{C} 10)$ \\
$s_{63}$ & $\omega(\mathrm{C} 3-\mathrm{N} 1-\mathrm{C} 4: \mathrm{C} 2)$ \\
$s_{64}$ & $\omega(\mathrm{N} 1-\mathrm{C} 3-\mathrm{C} 5: \mathrm{O} 13)$ \\
$s_{65}$ & $\omega(\mathrm{C} 7-\mathrm{C} 8-\mathrm{N} 9: \mathrm{H} 22)$ \\
$s_{66}$ & $\omega(\mathrm{N} 9-\mathrm{C} 10-\mathrm{C} 11: \mathrm{H} 23)$ \\
$s_{67}$ & $\omega(\mathrm{C} 8-\mathrm{C} 7-\mathrm{C} 12: \mathrm{C} 4)$ \\
$s_{68}$ & $\omega(\mathrm{C} 10-\mathrm{C} 11-\mathrm{C} 12: \mathrm{H} 24)$ \\
$s_{69}$ & $\omega(\mathrm{C} 7-\mathrm{C} 12-\mathrm{C} 11: \mathrm{H} 25)$ \\
\hline
\end{tabular}

a Abbreviations used: $r$, stretching; $\angle$, bending; $\omega$, out of plane bending; $\tau$, torsion. See Fig. 3 for the atom numbering. 
Table S3: The scaled harmonic force constants of the (eq, sp) conformer of cotinine a

\begin{tabular}{|c|c|c|c|c|c|c|c|c|c|c|c|c|c|c|c|c|c|}
\hline$i$ & $j$ & $F(i j)$ & $i$ & $j$ & $F(i j)$ & $i$ & $j$ & $F(i j)$ & $i$ & $j$ & $F(i j)$ & $i$ & $j$ & $F(i j)$ & $i$ & $j$ & $F(i j)$ \\
\hline 1 & 1 & 5.000 & 1 & 2 & 0.278 & 2 & 2 & 6.109 & 1 & 3 & 0.182 & 2 & 3 & 0.311 & 3 & 3 & 4.686 \\
\hline 2 & 4 & 0.350 & 4 & 4 & 3.682 & 3 & 5 & 0.271 & 5 & 5 & 3.658 & 1 & 6 & -0.139 & 2 & 6 & -0.108 \\
\hline 3 & 6 & -0.148 & 4 & 6 & 0.206 & 6 & 6 & 4.239 & 2 & 7 & 1.273 & 3 & 7 & -0.161 & 4 & 7 & 0.657 \\
\hline 5 & 7 & -0.120 & 7 & 7 & 11.776 & 1 & 8 & 0.291 & 8 & 8 & 4.990 & 9 & 9 & 4.795 & 10 & 10 & 4.912 \\
\hline 11 & 11 & 4.948 & 12 & 12 & 4.850 & 6 & 13 & 0.104 & 13 & 13 & 4.963 & 4 & 14 & 4.869 & 3 & 15 & 0.374 \\
\hline 5 & 15 & 0.263 & 15 & 15 & 4.011 & 3 & 16 & 0.180 & 16 & 16 & 4.680 & 17 & 17 & 7.768 & 18 & 18 & 6.417 \\
\hline 15 & 19 & 0.130 & 17 & 19 & 0.414 & 19 & 19 & 6.694 & 15 & 20 & 0.203 & 18 & 20 & 1.355 & 19 & 20 & -0.101 \\
\hline 20 & 20 & 6.063 & 15 & 21 & 0.208 & 19 & 21 & 0.162 & 21 & 21 & 7.274 & 15 & 22 & 0.209 & 18 & 22 & -0.882 \\
\hline 20 & 22 & 1.083 & 21 & 22 & -0.116 & 22 & 22 & 5.905 & 17 & 23 & 0.263 & 19 & 23 & 0.106 & 23 & 23 & 5.090 \\
\hline 18 & 24 & 0.286 & 20 & 24 & 0.125 & 24 & 24 & 5.085 & 25 & 25 & 5.214 & 21 & 26 & 0.123 & 26 & 26 & 5.191 \\
\hline 2 & 27 & 0.222 & 3 & 27 & -0.106 & 27 & 27 & 0.751 & 2 & 28 & 0.403 & 7 & 28 & 0.121 & 28 & 28 & 1.001 \\
\hline 4 & 29 & 0.155 & 6 & 29 & -0.256 & 29 & 29 & 0.600 & 30 & 30 & 0.592 & 12 & 31 & 0.151 & 31 & 31 & 0.660 \\
\hline 4 & 32 & -0.107 & 11 & 32 & 0.108 & 32 & 32 & 0.549 & 5 & 33 & -0.279 & 6 & 33 & 0.273 & 33 & 33 & 0.652 \\
\hline 34 & 34 & 0.647 & 14 & 35 & 0.124 & 35 & 35 & 0.727 & 5 & 36 & -0.117 & 6 & 36 & -0.106 & 13 & 36 & 0.113 \\
\hline 36 & 36 & 0.563 & 15 & 37 & 0.490 & 37 & 37 & 1.605 & 3 & 38 & 0.265 & 5 & 38 & -0.175 & 38 & 38 & 0.704 \\
\hline 3 & 39 & 0.344 & 5 & 39 & -0.151 & 15 & 39 & 0.257 & 37 & 39 & 0.764 & 39 & 39 & 1.413 & 5 & 40 & 0.162 \\
\hline 15 & 40 & 0.272 & 16 & 40 & -0.140 & 37 & 40 & 1.082 & 39 & 40 & 0.487 & 40 & 40 & 1.541 & 1 & 41 & 0.460 \\
\hline 41 & 41 & 0.617 & 42 & 42 & 0.777 & 10 & 43 & 0.119 & 43 & 43 & 0.785 & 9 & 44 & -0.144 & 44 & 44 & 0.560 \\
\hline 10 & 45 & 0.141 & 45 & 45 & 0.552 & 1 & 46 & -0.286 & 2 & 46 & -0.164 & 3 & 46 & -0.271 & 5 & 46 & -0.351 \\
\hline 6 & 46 & 0.576 & 7 & 46 & 0.331 & 15 & 46 & 0.105 & 28 & 46 & 0.120 & 46 & 46 & 1.697 & 2 & 47 & 0.136 \\
\hline 3 & 47 & -0.116 & 4 & 47 & -0.113 & 5 & 47 & 0.192 & 6 & 47 & -0.111 & 7 & 47 & -0.252 & 46 & 47 & -0.485 \\
\hline 47 & 47 & 1.285 & 17 & 48 & 0.329 & 19 & 48 & -0.172 & 48 & 48 & 0.581 & 18 & 49 & 0.273 & 20 & 49 & -0.120 \\
\hline 49 & 49 & 0.564 & 19 & 50 & 0.147 & 20 & 50 & 0.131 & 21 & 50 & -0.164 & 22 & 50 & -0.160 & 50 & 50 & 0.817 \\
\hline 19 & 51 & 0.113 & 21 & 51 & -0.127 & 22 & 51 & 0.110 & 51 & 51 & 0.488 & 22 & 52 & -0.215 & 52 & 52 & 0.502 \\
\hline 17 & 53 & 0.402 & 19 & 53 & -0.290 & 21 & 53 & 0.313 & 53 & 53 & 1.138 & 15 & 54 & -0.137 & 17 & 54 & 0.384 \\
\hline 23 & 54 & 0.117 & 53 & 54 & 0.515 & 54 & 54 & 1.129 & 15 & 55 & -0.171 & 18 & 55 & -0.292 & 22 & 55 & 0.274 \\
\hline 24 & 55 & 0.100 & 55 & 55 & 0.919 & 56 & 56 & 0.102 & 57 & 57 & 0.207 & 60 & 60 & 0.248 & 61 & 61 & 0.344 \\
\hline 62 & 62 & 0.275 & 63 & 63 & 0.125 & 64 & 64 & 0.636 & 65 & 65 & 0.325 & 66 & 66 & 0.331 & 67 & 67 & 0.388 \\
\hline 68 & 68 & 0.305 & 69 & 69 & 0.335 & & & & & & & & & & & & \\
\hline
\end{tabular}

a Units for energy, stretching and bending coordinates are aJ, $\AA$ and radians, respectively.

The force constants with the absolute values greater than 0.1 in these units are listed although all the force constants were used in normal coordinate calculations. All the force constants are available from the authors upon request. 
Table S4: $\quad r_{\mathrm{a}}$ Distances and mean amplitudes of the (ax, sc) conformer of cotinine ( $\AA$ )

\begin{tabular}{|c|c|c|c|c|c|}
\hline atom pair ${ }^{\mathrm{a}}$ & $r_{\mathrm{a}}$ & $l$ & atom pair & $r_{\mathrm{a}}$ & $l$ \\
\hline $\mathrm{C}-\mathrm{H}_{\text {pyridine }}$ & 1.097 & 0.077 & $\mathrm{C} 2 \cdots \mathrm{C} 7$ & 3.166 & 0.159 \\
\hline $\mathrm{C}-\mathrm{H}_{\text {alkyl }}$ & 1.103 & 0.078 & $\mathrm{C} 2 \cdots \mathrm{C} 12$ & 3.288 & 0.280 \\
\hline C3-013 & 1.218 & 0.038 & C5 $\cdots$ C7 & 3.290 & 0.194 \\
\hline C8-N9 & 1.340 & 0.045 & C6 $\cdots$ C8 & 3.348 & 0.228 \\
\hline N9-C10 & 1.343 & 0.045 & C3… 7 & 3.351 & 0.147 \\
\hline N1-C3 & 1.382 & 0.047 & C6‥C12 & 3.425 & 0.201 \\
\hline C11-C12 & 1.391 & 0.047 & C3‥C12 & 3.505 & 0.249 \\
\hline C10-C11 & 1.393 & 0.047 & $\mathrm{C} 4 \cdots \mathrm{O} 13$ & 3.528 & 0.062 \\
\hline C7-C12 & 1.396 & 0.047 & C6…013 & 3.589 & 0.075 \\
\hline C7-C8 & 1.397 & 0.047 & C5 $\cdots$ C12 & 3.642 & 0.268 \\
\hline N1-C2 & 1.456 & 0.050 & $\mathrm{~N} 1 \cdots \mathrm{C} 8$ & 3.721 & 0.125 \\
\hline N1-C4 & 1.461 & 0.051 & $\mathrm{C} 2 \cdots \mathrm{C} 5$ & 3.724 & 0.070 \\
\hline C4-C7 & 1.519 & 0.054 & $\mathrm{C} 2 \cdots \mathrm{C} 6$ & 3.753 & 0.081 \\
\hline C3-C5 & 1.530 & 0.055 & $\mathrm{C} 4 \cdots \mathrm{N} 9$ & 3.796 & 0.074 \\
\hline C5-C6 & 1.534 & 0.054 & C4 $\cdots$ C11 & 3.799 & 0.072 \\
\hline C4-C6 & 1.553 & 0.057 & $\mathrm{C} 4 \cdots \mathrm{C} 10$ & 4.225 & 0.074 \\
\hline C8*CC10 & 2.268 & 0.056 & $\mathrm{~N} 1 \cdots \mathrm{C} 11$ & 4.228 & 0.142 \\
\hline $\mathrm{N} 1 \cdots \mathrm{O} 13$ & 2.301 & 0.056 & $\mathrm{C} 2 \cdots \mathrm{C} 8$ & 4.273 & 0.325 \\
\hline N1ㄷㄷ & 2.336 & 0.059 & $\mathrm{C} 12 \cdots \mathrm{O} 13$ & 4.343 & 0.343 \\
\hline N1‥C6 & 2.360 & 0.067 & $\mathrm{C} 5 \cdots \mathrm{C} 8$ & 4.363 & 0.279 \\
\hline C3‥C4 & 2.373 & 0.060 & $\mathrm{C} 7 \cdots \mathrm{O} 13$ & 4.422 & 0.181 \\
\hline $\mathrm{C} 10 \cdots \mathrm{C} 12$ & 2.376 & 0.057 & C $2 \cdots \mathrm{C} 11$ & 4.479 & 0.276 \\
\hline C8…C12 & 2.385 & 0.058 & C6‥N9 & 4.557 & 0.223 \\
\hline C7‥C11 & 2.404 & 0.058 & С3‥C8 & 4.630 & 0.126 \\
\hline C4 $\cdots$ C5 & 2.414 & 0.072 & C6‥C11 & 4.631 & 0.179 \\
\hline C3‥C6 & 2.420 & 0.067 & C3‥C11 & 4.837 & 0.265 \\
\hline C7‥N9 & 2.424 & 0.057 & N1 ‥N9 & 4.841 & 0.123 \\
\hline N9..C11 & 2.426 & 0.057 & C5…C11 & 4.879 & 0.298 \\
\hline C2 $\cdots$ C3 & 2.476 & 0.071 & $\mathrm{~N} 1 \cdots \mathrm{C} 10$ & 4.987 & 0.117 \\
\hline C5 $\cdots$ O13 & 2.477 & 0.067 & C6 $\cdots$ C10 & 5.033 & 0.163 \\
\hline N1 ‥C7 & 2.480 & 0.075 & $\mathrm{C} 2 \cdots \mathrm{N} 9$ & 5.240 & 0.345 \\
\hline C4 $\cdots$ C12 & 2.533 & 0.072 & $\mathrm{C} 2 \cdots \mathrm{C} 10$ & 5.278 & 0.285 \\
\hline C4 $\cdots$ C8 & 2.543 & 0.075 & C5 $\cdots$ N9 & 5.430 & 0.320 \\
\hline $\mathrm{C} 2 \cdots \mathrm{C} 4$ & 2.551 & 0.078 & C5ㄷ.C10 & 5.594 & 0.312 \\
\hline C6 6 드 & 2.563 & 0.087 & $\mathrm{C} 11 \cdots \mathrm{O} 13$ & 5.600 & 0.383 \\
\hline C7 $\cdots$ C10 & 2.715 & 0.064 & C3 $\cdots$ N9 & 5.701 & 0.163 \\
\hline C8*..C11 & 2.721 & 0.064 & $\mathrm{C} 8 \cdots \mathrm{O} 13$ & 5.729 & 0.150 \\
\hline N9...C12 & 2.806 & 0.067 & C3 $\cdots$ C10 & 5.733 & 0.222 \\
\hline $\mathrm{C} 2 \cdots \mathrm{O} 13$ & 2.854 & 0.108 & 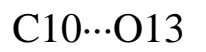 & 6.629 & 0.311 \\
\hline $\mathrm{N} 1 \cdots \mathrm{C} 12$ & 2.869 & 0.156 & N9...O13 & 6.732 & 0.213 \\
\hline
\end{tabular}

a See Fig. 3 for the atom numbering. Non-bonded $\mathrm{C} \cdots \mathrm{H}, \mathrm{N} \cdots \mathrm{H}, \mathrm{O} \cdots \mathrm{H}$ and $\mathrm{H} \cdots \mathrm{H}$ pairs are not listed although they were included in the data analysis. 
Table S5: The correlation matrix for cotinine ${ }^{a}$

\begin{tabular}{lccccccccccccc}
\hline & $k_{1}$ & $k_{2}$ & $x$ & $r_{1}$ & $r_{2}$ & $r_{3}$ & $r_{4}$ & $r_{5}$ & $r_{6}$ & $\theta_{1}$ & $\theta_{2}$ & $\theta_{3}$ & $\theta_{4}$ \\
\hline$k_{1}$ & 1.00 & & & & & & & & & & & & \\
$k_{2}$ & 0.29 & 1.00 & & & & & & & & & & & \\
$x$ & 0.01 & -0.03 & 1.00 & & & & & & & & & & \\
$r_{1}$ & 0.15 & 0.24 & 0.20 & 1.00 & & & & & & & & & \\
$r_{2}$ & -0.03 & -0.01 & -0.22 & -0.43 & 1.00 & & & & & & & & \\
$r_{3}$ & 0.28 & 0.25 & -0.25 & -0.38 & 0.22 & 1.00 & & & & & & & \\
$r_{4}$ & -0.22 & -0.03 & -0.07 & -0.06 & -0.05 & 0.23 & 1.00 & & & & & & \\
$r_{5}$ & -0.12 & -0.12 & 0.15 & 0.15 & -0.75 & 0.06 & 0.40 & 1.00 & & & & & \\
$r_{6}$ & -0.30 & -0.08 & 0.08 & 0.00 & 0.05 & -0.02 & 0.30 & 0.22 & 1.00 & & & & \\
$\theta_{1}$ & 0.04 & 0.08 & 0.44 & 0.09 & -0.23 & 0.16 & -0.04 & 0.25 & 0.02 & 1.00 & & & \\
$\theta_{2}$ & -0.11 & -0.08 & 0.18 & 0.19 & -0.35 & -0.55 & -0.39 & -0.06 & -0.08 & 0.02 & 1.00 & & \\
$\theta_{3}$ & 0.36 & 0.13 & 0.10 & 0.08 & -0.03 & 0.21 & 0.01 & 0.07 & 0.14 & 0.11 & -0.28 & 1.00 & \\
$\theta_{4}$ & -0.05 & -0.04 & 0.45 & 0.13 & -0.51 & -0.22 & -0.04 & 0.44 & 0.06 & 0.22 & 0.05 & -0.07 & 1.00 \\
$\theta_{5}$ & 0.05 & 0.00 & -0.02 & -0.04 & 0.18 & -0.06 & -0.13 & -0.23 & -0.01 & 0.10 & 0.33 & 0.01 & -0.34 \\
$\theta_{6}$ & 0.03 & -0.08 & 0.55 & 0.34 & -0.54 & -0.23 & -0.15 & 0.38 & 0.04 & 0.51 & 0.25 & 0.23 & 0.54 \\
$\phi_{1}$ & -0.17 & -0.10 & 0.17 & -0.03 & 0.00 & -0.15 & -0.01 & 0.01 & 0.00 & 0.05 & 0.03 & -0.32 & 0.10 \\
$\phi_{2}$ & -0.01 & 0.00 & 0.01 & 0.06 & -0.04 & -0.09 & -0.07 & -0.05 & -0.02 & 0.09 & 0.19 & -0.08 & 0.03 \\
\hline & & & & & & & & & & & & & \\
\end{tabular}

\begin{tabular}{lllll}
\hline & $\theta_{5}$ & $\theta_{6}$ & $\phi_{1}$ & $\phi_{2}$ \\
\hline$\theta_{5}$ & 1.00 & & & \\
$\theta_{6}$ & 0.27 & 1.00 & & \\
$\phi_{1}$ & -0.23 & -0.03 & 1.00 & \\
$\phi_{2}$ & 0.13 & 0.13 & -0.04 & 1.00 \\
\hline
\end{tabular}

a See Table 4 for the definitions of the structural parameters. $k_{1}$ and $k_{2}$ are the indices of resolution for the long and short camera distances, respectively. $x$ is the molar fraction of the (ax, sc) conformer. 